\title{
OPEN Orexin system is expressed in avian liver and regulates hepatic lipogenesis via ERK1/2 activation
}

\author{
E. S. Greene ${ }^{1}$, M. Zampiga ${ }^{2}$, F. Sirri ${ }^{2}$, T. Ohkubo ${ }^{3} \&$ Sami Dridi $^{1 凶}$
}

Orexins are originally characterized as orexigenic hypothalamic neuropeptides in mammals. Subsequent studies found orexin to be expressed and perform pleiotropic functions in multiple tissues in mammals. In avian (non-mammalian) species, however, orexin seemed to not affect feeding behavior and its physiological roles are poorly understood. Here, we provide evidence that orexin and its related receptors are expressed in chicken hepatocytes. Double immunofluorescence staining showed that orexin is localized in the ER, Golgi, and in the lysosomes in LMH cells. Brefeldin A treatment reduced orexin levels in the culture media, but increased it in the cell lysates. Administration of recombinant orexins upregulated the expression of orexin system in the liver of 9-day old chicks, but did not affect feed intake. Recombinant orexins increased fatty acid synthase (FASN) protein levels in chicken liver, activated acetyl-CoA carboxylase (ACC $\alpha$ ), and increased FASN, ATP citrate lyase(ACLY), and malic enzyme (ME) protein expression in LMH cells. Blockade ERK1/2 activation by PD98059 attenuated these stimulating effects of orexin on lipogenic factors. Overexpression of ERK1/2 increased the expression of lipogenic genes, and orexin treatment induced

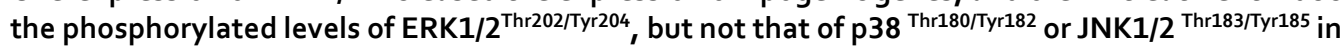
chicken liver and LMH cells. Taken together, this is the first report evidencing that orexin is expressed and secreted from chicken hepatocytes, and that orexin induced hepatic lipogenesis via activation of ERK1/2 signaling pathway.

Orexin $\mathrm{A}$ and $\mathrm{B}^{1}$ (also known as hypocretins 1 and $2^{2}$ ) are 33-amino acid (ORX-A) and 28-amino acid (ORX-B) peptides derived from proteolytic cleavage of a single precursor, prepro-orexin, and were originally discovered to be produced by several feeding-related hypothalamic nuclei in rodents and humans ${ }^{1,2}$. More recently, orexins and their cognate receptors have been found to be expressed in several mammalian peripheral tissues, including the gastrointestinal tract, pancreas, adrenals, kidney, adipose tissue, and the reproductive tract (for review $\left.\mathrm{see}^{3}\right)$, suggesting potential pleiotropic functions and involvement in multiple versatile physiological processes (for review see ${ }^{4}$.

The physiological effects of orexin (ORX) are mediated by two G-protein coupled receptors, orexin receptor 1 (ORXR1) and orexin receptor 2 (ORXR2). Originally identified as regulators of feeding behavior ${ }^{5}$, orexins are also known to control sleep-wake cycle ${ }^{6-8}$, energy balance and glucose homeostasis ${ }^{9,10}$, autonomic function ${ }^{11,12}$, and as a modulator of the stress response $\mathrm{e}^{13,14}$. In adipocytes, orexin has been shown to decrease lipolysis ${ }^{15}$ and increase lipid accumulation ${ }^{16}$, hinting at effects on adipogenesis and lipogenesis.

In avian (non-mammalian) species, although prepro-orexin sequence is highly conserved ${ }^{17}$, and avian orexin system was found in the brain, testis, ovary, stomach, and intestine ${ }^{18,19}$, little is known about its physiological roles. Recently, we have shown the presence of ORX and its receptors, ORXR1 and ORXR2, in the muscle ${ }^{20}$ of avian species and demonstrated its role in modulating energy metabolism and mitochondrial function.

As orexin has been shown to play a key role in lipid metabolism in rodents, and as fat accumulation and liver hemorrhagic syndrome are a source of substantial burden and cost to poultry, we undertook this study to determine the role of orexin in avian lipid metabolism. Since the liver is the primary site for de novo fatty acid synthesis in chicken ${ }^{21}$, we hypothesized that orexin may be produced by the hepatocytes and may regulate lipogenesis. Our data showed that orexin system is expressed in chicken liver and avian hepatocyte cell lines and that orexin administration modulate the expression of lipogenic genes. The effect of orexin on avian hepatic lipogenesis seems to be mediated via extracellular signal-regulated kinase 1/2 (ERK1/2) signaling pathways.

${ }^{1}$ Center of Excellence for Poultry Science, University of Arkansas, 1260 W. Maple Street, Fayetteville, AR 72701, USA. ${ }^{2}$ Dipartimento Di Scienze E Tecnologie Agro-Alimentari, Alma Mater Studiorum-Università Di Bologna, Bologna, Italy. ${ }^{3}$ College of Agriculture, Ibaraki University, Ibaraki 300-0393, Japan. ${ }^{\varpi}$ email: dridi@uark.edu 


\section{Materials and methods}

Animals. Day-old male broilers (Cobb 500, Cobb-Vantress, Siloam Springs, AR) were divided into 3 weightmatched groups $(39.75 \pm 0.26 \mathrm{~g})$, reared for 8 days, and have ad libitum access to clean water and feed $(4.090$ Mcal ME kg-1 and $22 \% \mathrm{CP}$ ). The ambient temperature was reduced gradually ( ${ }^{\circ} \mathrm{C}$ every 2 days) from 32 to $29^{\circ} \mathrm{C}$, and the relative humidity was maintained at $55 \pm 2 \%$. Feed and water intake were recorded hourly. On day 9, birds received an intraperitoneal (i.p.) injection of recombinant human orexin-A or -B (rORX-A or -B; Alpha Diagnostic International, San Antonio, TX, $100 \mathrm{ng} / 100 \mathrm{~g} \mathrm{BW}, \mathrm{n}=12$ ). The control group received a saline solution. Three hours after administration, birds were euthanized by cervical dislocation. Blood was collected and $250 \mu \mathrm{L}$ added to Trizol LS reagent (Life Technologies, Carlsbad, CA) for mRNA extraction. Liver samples were immediately collected, snap frozen in liquid nitrogen and stored at $-80^{\circ} \mathrm{C}$ for further analysis. The present study was conducted in accordance with the recommendations in the guide for the care and use of laboratory animals of the National Institutes of Health and the protocols were approved by the University of Arkansas Institutional Animal Care and Use Committee under protocol \# 16084.

Leghorn Male Hepatoma (LMH) cell culture and treatments. LMH cells (ATCC CRL-2117) or spontaneously immortalized chicken embryonic hepatocytes $\left(\mathrm{siCEH}^{22}\right)$ were grown on $0.1 \%$ gelatin-coated culture dishes in Waymouth's media (Life Technologies, Carlsbad, CA), supplemented with 10\% FBS (Life Technologies, Carlsbad, CA), and $1 \%$ penicillin-streptomycin (BioBasic, Amherst, NY) at $37^{\circ} \mathrm{C}$, in a humidified atmosphere of $5 \% \mathrm{CO}_{2}$ and $95 \%$ air. At $~ 80 \%$ confluence, cells were synchronized with serum-free media overnight and treated with different doses $(0,10$, and $100 \mathrm{nM})$ of recombinant human ORX-A or ORX-B (Alpha Diagnostic International, San Antonio, TX) for $24 \mathrm{~h}$. The dose of orexins was selected based on pilot and previous studies ${ }^{16,20,23,24} .30$ min before orexin treatments, $\mathrm{LMH}$ cells were incubated with brefeldin A $(0.3 \mu \mathrm{g} / \mathrm{mL})$ for $24 \mathrm{~h}$ and lysates and medium were subjected to immunoblot analyses. All culture experiments were performed with cells from passages $10-15$ and repeated at least twice.

Transient overexpression and inhibition of ERK1/2. At 50-60\% confluence, LMH cells were pretreated with PD98059 (10 $\mu$ M, ThermoFisher Scientific, Waltham, MA) 30 min before orexin administration. Cells were transiently transfected with pEGFP-ERK1, pEGFP-ERK2, and pEGFP empty vector (mock) using Lipofectamine 2000 (ThermoFisher Scientific, Waltham, MA) according to the manufacturer's instructions. Six to eight hours post transfection, complete medium was added and cells were maintained for additional $16 \mathrm{~h}$ and were processed for immunoblotting.

RNA isolation and quantitative real-time PCR. For chicken liver and cell lysates, total RNA was extracted using Trizol reagent (Life Technologies, Carlsbad, CA) according to the manufacturer's instructions, and concentration and quality were determined by using the Take3 micro-volume plate and the Synergy HT multimode microplate reader (BioTek, Winooski, VT). The ratio of absorbance (A260/280) as well as agarose gel electrophoresis were used to assess RNA integrity and quality. RNA was reverse transcribed using qScript cDNA Sythesis Supermix (Quanta Biosciences, Gaithersburg, MD), and amplified by qPCR (Applied Biosystems 7500 Real Time System) with SYBR green master mix (Life Technologies, Carlsbad, CA) as previously described ${ }^{25-27}$. Relative expression of the target genes was determined using the $2^{-\Delta \Delta C T}$ method, with normalization to $r 18 S$ expression $^{28}$. Oligonucleotide primers specific for chicken are summarized in Table 1.

Conventional PCR. Total RNA was extracted and reverse transcribed as described above. Fragments of ORX, ORXR1/2, and $\mathrm{r} 18 S$ were amplified by PCR using specific primers ${ }^{20}$. PCR was performed using $43 \mu \mathrm{L}$ of Platinum PCR SuperMix (Life Technologies, Carlsbad, CA), $5 \mu \mathrm{L}$ of cDNA, and $1 \mu \mathrm{L}$ of each forward and reverse primers. Thermal cycling parameters for ORX and ORXR $1 / 2$ were: $94{ }^{\circ} \mathrm{C}$ for $4 \mathrm{~min}$, followed by 39 cycles of $94^{\circ} \mathrm{C}$ for $30 \mathrm{~s}, 50{ }^{\circ} \mathrm{C}$ for $30 \mathrm{~s}, 72{ }^{\circ} \mathrm{C}$ for $1 \mathrm{~min}$, with a final extension at $72{ }^{\circ} \mathrm{C}$ for $10 \mathrm{~min}$. For $\mathrm{r} 18 \mathrm{~S}$, the temperatures were the same, but the annealing and elongation steps were each held for $1 \mathrm{~min}$. Fragments were separated on a $1 \%$ agarose gel electrophoresis and images captured using the FluorChem M MultiFluor System (ProteinSimple, San Jose, CA ).

Western blot analysis. Liver tissue and cells were homogenized in lysis buffer (10 mM Tris Base, $\mathrm{pH}$ 7.4, $150 \mathrm{mM} \mathrm{NaCl}, 1 \mathrm{mM}$ EDTA, $1 \mathrm{mM}$ EGTA, 0.1\% Triton X-100, 0.5\% NP-40, protease and phosphatase inhibitors). Protein concentrations were determined using a Bradford assay kit (Bio-Rad, Hercules, CA) and a Synergy HT multimode microplate reader (BioTek, Winooski, VT). Proteins ( $80 \mu \mathrm{g}$ liver tissue, $40 \mu \mathrm{g}$ cell lysate) were run on $4-12 \%$ gradient Bis-Tris gels (Life Technologies, Carlsbad, CA), and transferred to PVDF membranes. The membranes were blocked with 5\% non-fat milk in TBS-T for $1 \mathrm{~h}$ at room temperature, then incubated with primary antibodies (1:500-1:1000 dilution) overnight at $4{ }^{\circ} \mathrm{C}$. Secondary antibodies (1:5000) were diluted in $5 \%$ milk in TBS-T and membranes were incubated at room temperature for $1 \mathrm{~h}$. Primary antibodies used were: rabbit anti-ORX, rabbit anti-ORXR1, rabbit anti-ORXR2 (Interchim, France), rabbit anti-phospho-ACCa ${ }^{\text {Ser79, }}$, rabbit anti-ACCa, rabbit anti-FASN, rabbit anti-phospho-ERK1/2 Thr202/Tyr204, rabbit anti-ERK1/2, rabbit anti-

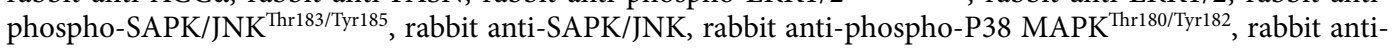
P38 MAPK (Cell Signaling Danvers, MA), and rabbit anti-nucleoline (Santa Cruz Biotechnology, Dallas, TX ). To assess protein loading, the expression of the housekeeping GAPDH protein was determined using rabbit antiGAPDH antibody (Santa Cruz Biotechnology, Dallas, TX). The signal was visualized by chemiluminescence (ECL Plus, GE Healthcare, Pittsburg, PA) and captured by the FluorChem M MultiFluor System (ProteinSimple, San Jose, CA). 


\begin{tabular}{|c|c|c|c|c|}
\hline Gene & Accession number ${ }^{\mathrm{a}}$ & Primer sequence & Orientation & Product size (bp) \\
\hline \multirow{2}{*}{ ORX } & \multirow{2}{*}{ AB056748 } & CCAGGAGCACGCTGAGAAG & For & \multirow{2}{*}{67} \\
\hline & & \begin{tabular}{|l} 
CCCATCTCAGTAAAAGCTCTTTGC \\
\end{tabular} & Rev & \\
\hline \multirow{2}{*}{ ORXR1 } & \multirow{2}{*}{ NM_001024584.1 } & TGCGCTACCTCTGGAAGGA & For & \multirow{2}{*}{58} \\
\hline & & GCGATCAGCGCCCATTC & Rev & \\
\hline \multirow{2}{*}{ ORXR2 } & \multirow{2}{*}{ XM_004945362 } & AAGTGCTGAAGCAACCATTGC & For & \multirow{2}{*}{61} \\
\hline & & AAGTGCTGAAGCAACCATTGC & Rev & \\
\hline \multirow{2}{*}{ ACC } & \multirow{2}{*}{ NM_205505.1 } & CAGGTATCGCATCACTATAGGTAACAA & For & \multirow{2}{*}{74} \\
\hline & & GTGAGCGCAGAATAGAAGGATCA & Rev & \\
\hline \multirow{2}{*}{ FAS } & \multirow{2}{*}{ NM_205155.2 } & ACTGTGGGCTCCAAATCTTCA & For & \multirow{2}{*}{70} \\
\hline & & CAAGGAGCCATCGTGTAAAGC & Rev & \\
\hline \multirow{2}{*}{$\mathrm{ME}$} & \multirow{2}{*}{ NM_204303.1 } & AGATGAAGCTGTCAAAAGGATATG & For & \multirow{2}{*}{62} \\
\hline & & CACGCCССTTCACTATCGA & Rev & \\
\hline \multirow{2}{*}{ ACLY } & \multirow{2}{*}{ NM_001030540.1 } & CTTTTAAGGGCATTGTTAGAGCAAT & For & \multirow{2}{*}{65} \\
\hline & & CCTCACCTCGTGCTCTTTCAG & Rev & \\
\hline \multirow{2}{*}{ SCD-1 } & \multirow{2}{*}{ NM_204890.1 } & CAATGCCACCTGGCTAGTGA & For & \multirow{2}{*}{52} \\
\hline & & CGGCCGATTGCCAAAC & Rev & \\
\hline \multirow{2}{*}{ SREBP-1 } & \multirow{2}{*}{ NM_204126.2 } & CATCCATCAACGACAAGATCGT & For & \multirow{2}{*}{82} \\
\hline & & CTCAGGATCGCCGACTTGTT & Rev & \\
\hline SRFRP, & XM 0152800372 & GCCTCTGATTCGGGATCACA & For & 63 \\
\hline SREBP-L & XM_01528905/.2 & \begin{tabular}{|l} 
GCTTCCTGGCTCTGAATCAATG \\
\end{tabular} & $\operatorname{Rev}$ & 63 \\
\hline PPARg & NM 0010014641 & CAAACCAACCATCCTGACGAT & For & 64 \\
\hline PPAR & NM_001001464.1 & GGAGGTCAGCCATTTTTTTGGA & Rev & 64 \\
\hline $\mathrm{PDAP}$ & NM 0010014601 & CACTGCAGGAACAGAACAAAGAA & For & 67 \\
\hline PFАKY & Nold_001001400.1 & TCCACAGAGCGAAACTGACATC & Rev & $0 \%$ \\
\hline INSIG 1 & & TGGCGCTGGTGCTGAAC & For & 63 \\
\hline INSIGI & & TGACCTCGTCGGGAAACAG & Rev & 63 \\
\hline INSSC, & NM 0013054651 & CAGCGCTAAAGTGGATTTTGC & For & 65 \\
\hline INSIGL & NM_001305465.1 & CAATTGACAGGGCTGCTAACG & Rev & 65 \\
\hline SCAP & XM 025147369 & TGGCCCAGAGACTCATCATG & For & 67 \\
\hline SCAP & XM_02514/369.1 & GCAGGATCCGTATAAACCAGGAT & Rev & $5 \%$ \\
\hline FRK & NM 2041501 & CGGACCATGATCACACAGGAT & For & 63 \\
\hline ERK & NM__204150.1 & CAGGAGCCCTGTACCAACGT & Rev & 63 \\
\hline INK & NM 2050951 & GCCGATGATCAGCCAGGAT & For & 60 \\
\hline 促 & 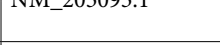 & GGCCCAATGGAAGCAAGAG & $\operatorname{Rev}$ & 02 \\
\hline$n 38$ & XM 4192635 & AGCTGGAGATTGAGGAATGGAA & For & 62 \\
\hline p38 & XM_419263.5 & CGGTGGCACAAAGCTGATTA & Rev & 62 \\
\hline $10^{\circ}$ & $\mathrm{AF} 173612$ & TCCCCTCCCGTTACTTGGAT & For & 60 \\
\hline $18 \mathrm{~s}$ & AF173612 & GCGCTCGTCGGCATGTA & Rev & 60 \\
\hline
\end{tabular}

Table 1. Oligonucleotide qPCR primers. ${ }^{a}$ Accession number refer to Genbank (NCBI).

Immunofluorescence. Immunofluorescence was performed as previously described ${ }^{25}$. Briefly, cells were grown in chamber slides and fixed with methanol for $10 \mathrm{~min}$ at $-20^{\circ} \mathrm{C}$, then permeabilized with Triton-X 100 . Cells were blocked with serum-free protein block (Dako, Carpinteria, CA) for $1 \mathrm{~h}$ at room temperature, then incubated with anti-ORX, anti-ORXR1, anti-ORXR2, anti-phospho-ACC ${ }^{\text {Ser79 }}$ or anti-phospho-ERK1/2 ${ }^{\text {Thr202/ }}$

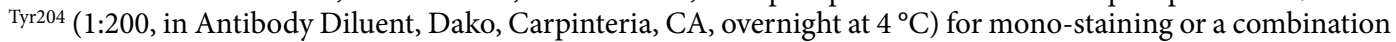
of anti-ORX with anti-ERGIC53 or anti-TGN38 for double-labeling immunofluorescence. For ER or lysosome co-labeling with ORX, cells were incubated with $100 \mathrm{nM}$ of ER-Tracker or Lyso-Tracker (ThermoFisher Scientific, Waltham, MA) for 15-30 min before fixation and then proceeded as mentioned above. Signal was visualized with DyLight 488-conjugated secondary antibody (Thermo Fisher Scientific, Grand Island, NY). Slides were cover slipped with Vectashield with DAPI (Vector Laboratories, Burlingame, CA), and images were obtained and analyzed using Zeiss Imager M2 and AxioVision software (Carl Zeiss Microscopy).

Statistical analysis. Data were analyzed by one-way ANOVA. Significant differences among individual group means were determined by Student-Newman-Keul's multiple range test with GraphPad Prism v. 6.00 (La Jolla, CA). Significance was set at $\alpha=0.05$. All data are represented as means \pm SEM. 


\begin{abstract}
Results
Orexin system is expressed in avian liver cells. By using immunoblot with antibody that cross-reacts with chicken orexin system ${ }^{20}$, we amplified a strong signal of orexin with only one band at the predicted size $(16 \mathrm{kDa})$ in chicken liver as well as in LMH and siCEH cells (Fig. 1a). However, we observed two bands with molecular weight of 16 and $230 \mathrm{kDa}$ in the hypothalamus (positive control, Fig. 1a). We amplified also a strong signal of orexin receptors in chicken hepatocytes (Fig. 1a). Orexin protein levels are significantly higher in the chicken liver compared to the hypothalamus (Fig. 1b), however its mRNA abundance are significantly lower (Fig. 1d). The expression (mRNA and protein) of orexin receptor (ORXR2) did not differ between the chicken liver and the hypothalamus (Fig. 1c,e,f). Immunofluorescence staining showed a strong reactivity of ORX and its related receptors (ORXR1 and ORXR2) in the cytoplasm of LMH cells (Fig. 1g). The predominant cytoplasmic localization of chicken orexin system was further confirmed by cell fractionation and Western blot analysis (Fig. 1h). To further determine the sub-cellular localization and distribution of orexin in LMH cells, doublelabeling immunofluorescence was performed. Chicken orexin was stained in combination with the well characterized ER fluorescent tracking dye, ER-Tracker, and shows to be predominantly localized in the ER of LMH cell line (yellow arrows, Fig. 2a-c). Immunofluorescence localization of Golgi markers suggests that chicken orexin is also localized, in the cis side (co-staining with ERGIC 53), and in the trans side of Golgi complex (co-staining with TGN38) in LMH cells (yellow arrows, Fig. 2d-i). The co-staining of chicken orexin with the lysosome tracking dye, lyso-Tracker deep red, suggests that chicken orexin is also localized in the lysosome (yellow arrows, Fig. 2j-1). Analysis of conditioned medium by Western blot showed specific secretion of orexin in LMH cells at $48 \mathrm{~h}$ (Fig. $2 \mathrm{~m}-\mathrm{o}$ ), and treatment of cells with brefeldin A (BFA, $0.3 \mu \mathrm{g} / \mathrm{mL}$ ), partially but significantly blocked orexin secretion which is demonstrated by a $\sim 26 \%$ decrease of ORX levels in the medium and a significant accumulation in cell lysate (Fig. 2p,q).
\end{abstract}

Orexin modulates its own expression in chicken liver and LMH cells. Intraperitoneal (IP) administration of recombinant orexin $\mathrm{B}$, but not orexin $\mathrm{A}$, significantly increases ORX mRNA abundances in chick blood (Fig. 3a). Neither recombinant orexin A nor B affect body weight (BW), feed intake (FI), or water consumption (WI) in chicks (Fig. 3b-d). Hepatic ORX and ORXR2 protein levels were significantly increased following administration of recombinant orexins (ORX-A and ORX-B) (Fig. 3e,f). Although, neither hepatic ORX nor ORXR1 mRNA abundances were affected by recombinant orexin treatments, hepatic ORXR2 gene expression was significantly down regulated by rORX-B administration (Fig. 3g). In LMH cells, treatment for $24 \mathrm{~h}$ with $100 \mathrm{nM}$ of rORX-A significantly down regulated prepro-ORX and ORXR1, but increased ORXR2 protein levels (Fig. 3h,i). Low dose of rORX-A increased both ORXR1 and ORXR2 protein expression and upregulated ORX and ORXR1 gene expression in LMH cells (Fig. $3 \mathrm{~h}-\mathrm{j}$ ). Treatment with rORX-B at high dose, however, increased ORX and ORXR1 protein levels and down regulated ORX mRNA abundance in LMH cells (Fig. 3k-m). Recombinant ORX-B at low dose (10 nM) induced both ORXR1 and ORXR2 protein expression and only ORXR2 mRNA levels in LMH cells (Fig. 3k-m).

Orexin regulates lipogenic program in chicken hepatocytes. Administration of recombinant orexin A or B significantly decreased phosphorylated $\mathrm{ACCa} a^{\mathrm{Ser} 79}$ and increased FASN protein levels, the rate-limiting enzymes involved in lipogenesis (Fig. $4 \mathrm{a}-\mathrm{c}$ ) and down regulated the expression of $A C C \alpha, F A S N, A C L Y$, and SCD1 genes in chicken liver (Fig. 4d,e). Recombinant orexin A significantly upregulated the hepatic expression of the transcription factors SREBP-1 and SREBP-2 (Fig. 4f), however recombinant orexin B significantly increased only SREBP-2 mRNA abundances (Fig. 4g). In vitro studies using LMH cells showed that both recombinant orexins at low and high dose significantly reduced the phosphorylated levels of ACCa at Ser79 site (Fig. 5a-c). As shown in Fig. 5d, immunofluorescence staining confirmed the reduced levels of ACCa phosphorylation in LMH cells following recombinant orexin B treatment. Orexin treatments also significantly increased the protein levels of FASN, ACLY, and ME in LMH cells (Fig. 5a-c). At transcriptional levels, recombinant orexin A down regulated $A C C \alpha$ and upregulated $M E$ and $S C D-1$ gene expression in LMH cells (Fig. 5e). Administration of high dose of recombinant orexin B significantly increased the mRNA levels of $A C C \alpha, F A S N, A C L Y$, and $M E$ (Fig. 5f). These changes were accompanied with a significant down regulation of SREBP-1 and an upregulation of SREBP-2, $I N S I G-1$, and PPAR $\gamma$ with recombinant orexin A treatment, and an upregulation of SREBP-1 and a down regulation of INSIG-1 and SCAP with the administration of recombinant orexin B (Fig. $5 \mathrm{~g}, \mathrm{~h}$ ).

Orexin B modulates hepatic lipogenesis via ERK1/2 pathway. Treatment with both doses of recom-

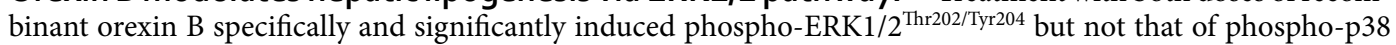
Thr180/Tyr182 or phospho-JNK1/2 Thr183/Tyr185 in LMH cells (Fig. 6a-c). High dose of recombinant orexin B significantly up regulated the expression of ERK1, ERK2, JNK, and P38 genes in LMH cells (Fig. 6e). Administration of recombinant orexin $\mathrm{A}$, however, did not elicit any changes to ERK1/2, p38, or JNK1/2 at both mRNA and protein levels (Fig. 6a,b,d). Overexpression of ERK1 or ERK2 (Fig. 6f,g) significantly decreased phospho-ACC ${ }^{\text {Ser79 }}$ and increased ME protein levels in LMH cells (Fig. 6f,g). ERK1 and ERK2 overexpression significantly upregulated the expression of ACCa, FASN, ACLY, and SREBP-1 (Fig. 6h). Pharmacological inhibition of ERK1/2 activation by PD98059 (Fig. 7a,b) blockaded the effects of recombinant orexin B on lipogenic program in LMH cells (increased phospho-ACCa ${ }^{\text {Ser79 }}$ and decreased FASN protein levels and down regulated ACC $\alpha$ and ME mRNA abundances) (Fig. 7c-e). Recombinant orexins specifically activated ERK1/2 at Thr202/Tyr204 site, but not P38 or JNK, in chicken liver (Fig. 8a-c). Recombinant orexins did not affect the hepatic expression of ERK1/2, P38, or JNK1/2 genes (Fig. 8d,e). 


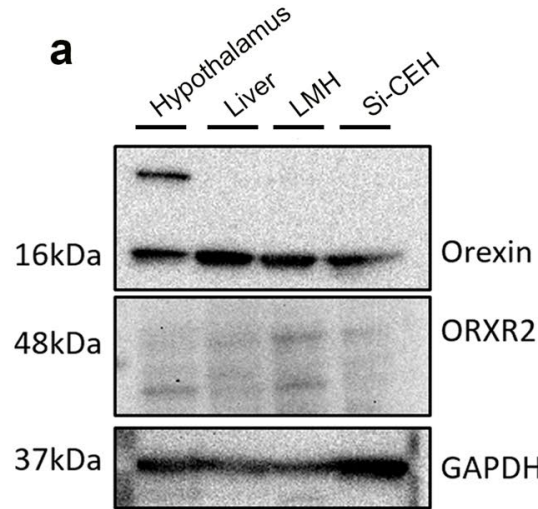

g
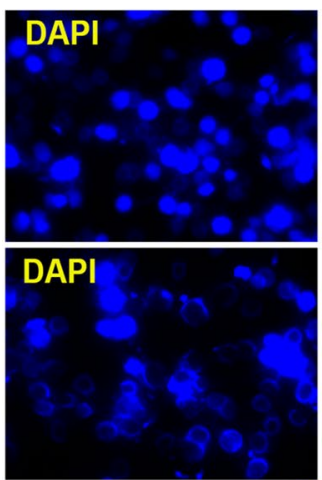

\section{DAPI}

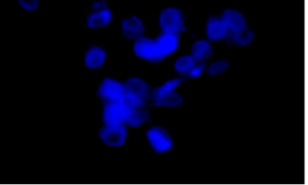

b

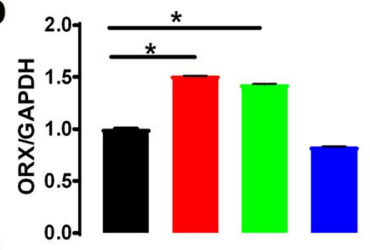

C
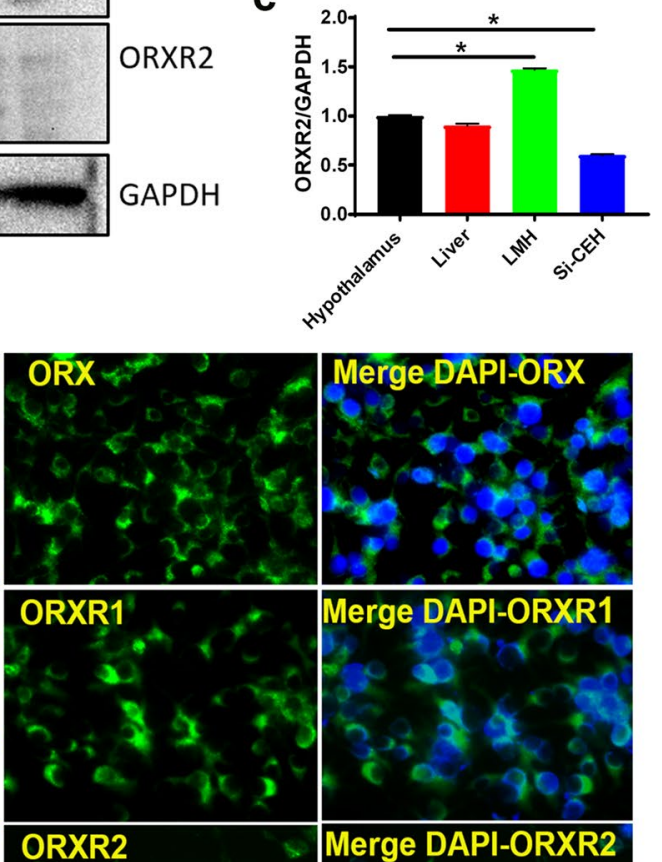

d

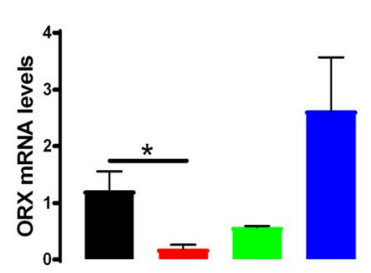

e
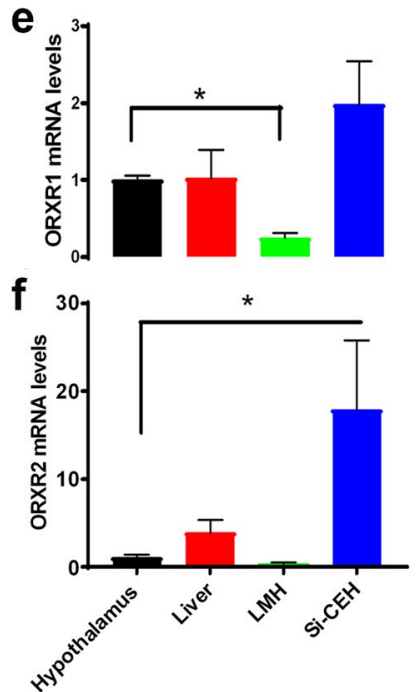

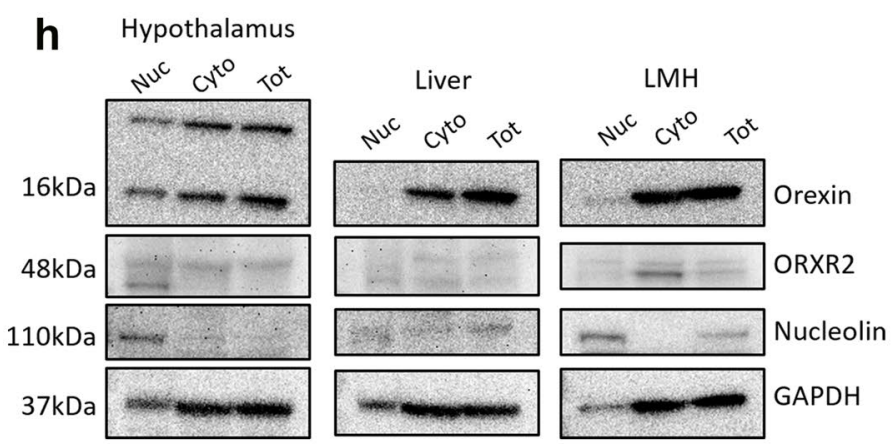

Figure 1. Orexin and its related receptors are expressed in chicken liver and hepatocyte cells. (a-c) Total proteins were isolated from chicken hypothalamus (positive control), liver, LMH, and siCEH cells and subjected to Western blot using anti-orexin and anti-ORXR2 antibodies and data are expressed as ratios to GAPDH. (d-f) Total RNA was reverse transcribed and subjected to RT-qPCR as described in the "Materials and methods" with chicken hypothalamus served as a calibrator, and relative expression was determined by $2^{-\Delta \Delta \mathrm{Ct}} \operatorname{method}^{28}$. $(\mathbf{g})$ Distribution of intracellular orexin system was determined by immunofluorescence staining with secondary antibody conjugated with AlexaFluor 488 (green) and DAPI (blue). (h) Proteins of cellular components (nucleus, cytoplasmic, and total) were obtained by cell fractionation and submitted to Western blot analysis as described above. Nucleolin was used as a positive control for the nuclear fraction. ${ }^{\star}$ Significant difference from the hypothalamus, $P<0.05$. Cyto cytoplasmic, $L M H$ leghorn male hepatoma cells, Nuc nuclear, siCEH spontaneously immortalized chicken embryonic hepatocytes, Tot total. Full-length blots/gels are presented in Supplementary Fig. S1. 

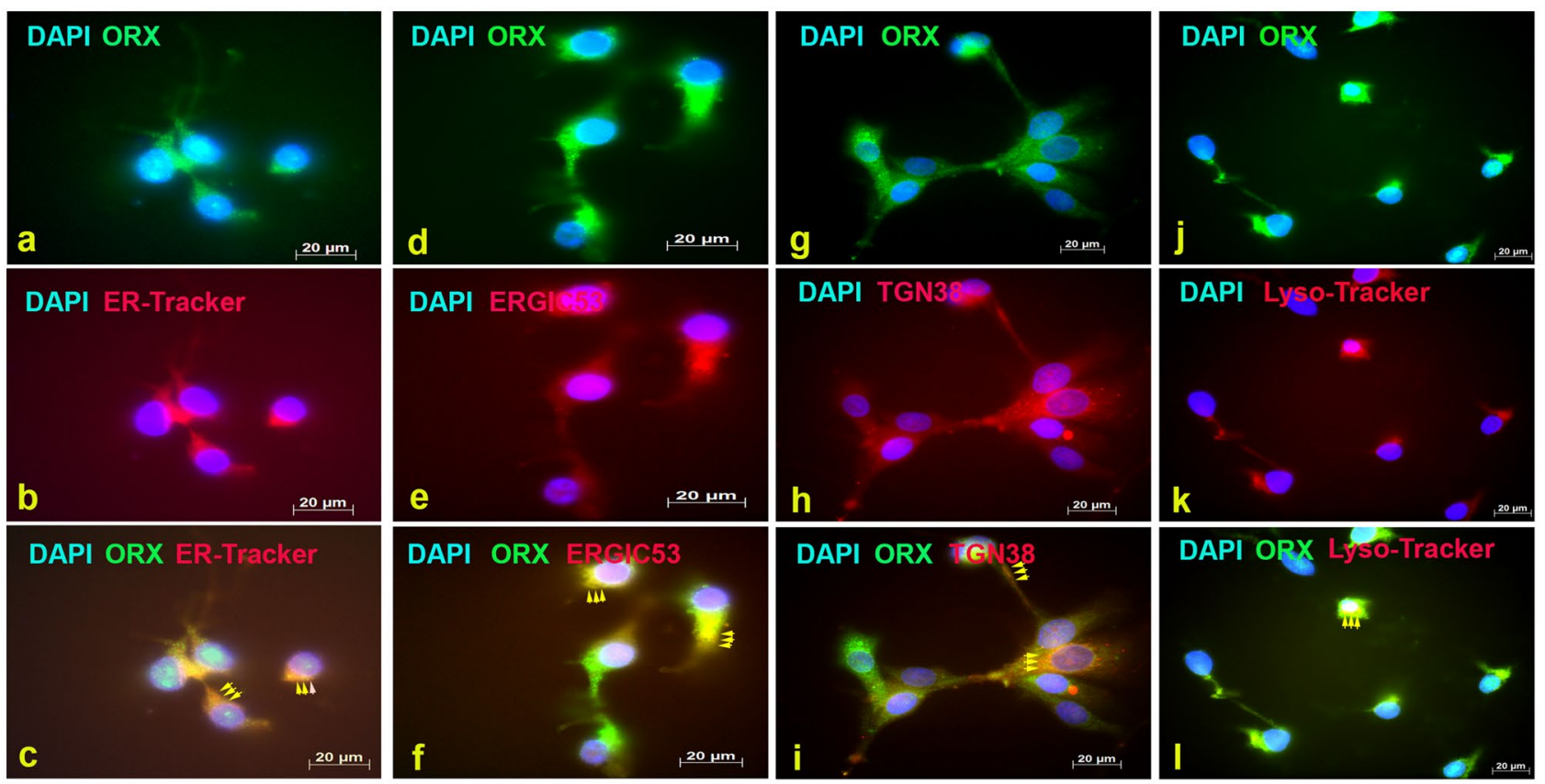

m

$\mathbf{n}$ Media

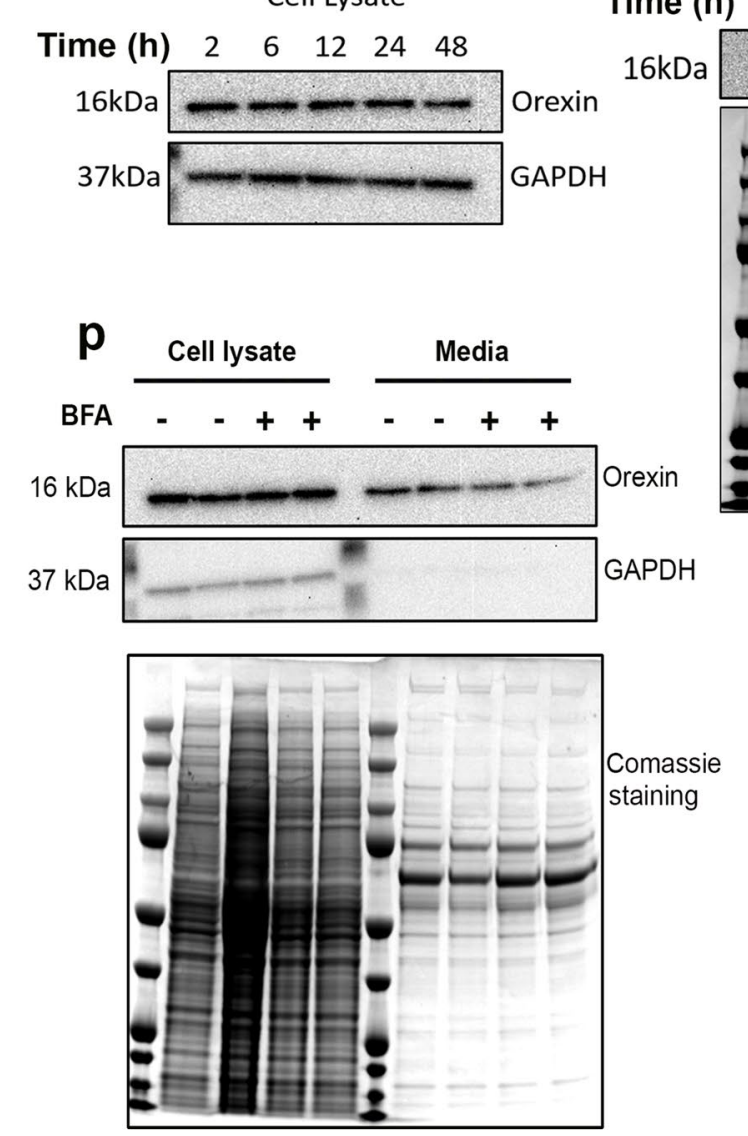

Time (h) $2 \quad 6 \quad 12 \quad 24 \quad 48$

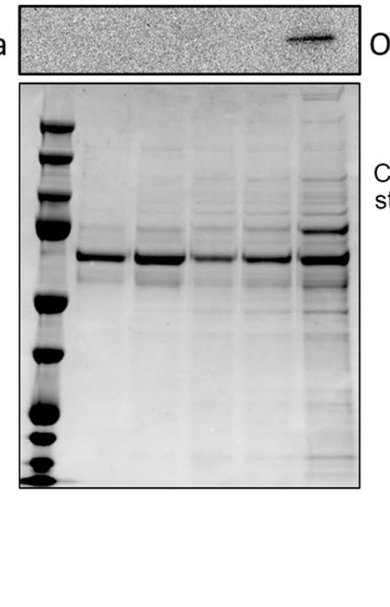

Orexin

Comassie staining
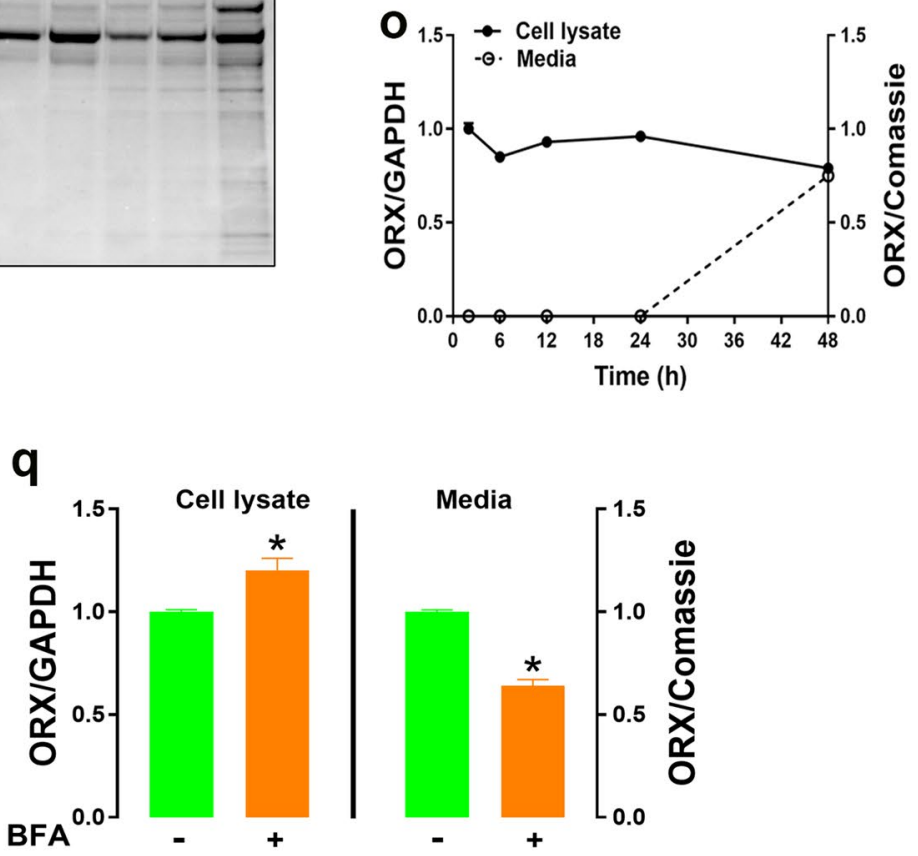

Figure 2. Subcellular localization and secretion of orexin by LMH cells. Chicken orexin was stained in combination with well characterized markers of the ER (ER-Tracker, $\mathbf{a}-\mathbf{c}$ ), cis (ERGIC53, $\mathbf{d}-\mathbf{f}$ ) and trans side (TGN38, $\mathbf{g}-\mathbf{i}$ ) of the Golgi complex, and the lysosome (lyso-Tracker, $\mathbf{j}-\mathbf{l}$ ). Yellow arrows showed the co-localization of chicken orexin (ORX) with target organelle-markers. Cell monolayers were incubated in serum-free media for indicated time periods, and collected media and cell lysates were subject to Western blot for orexin protein expression $(\mathbf{m}-\mathbf{o})$. Cells were pretreated with brefeldin A and collected media and cell lysates were subjected to Western blot for orexin protein expression (p,q). Full-length blots/gels are presented in Supplementary Fig. S1. 
a

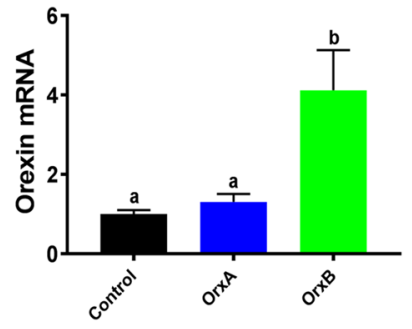

b

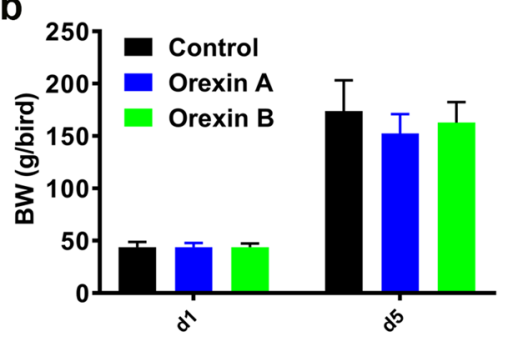

e

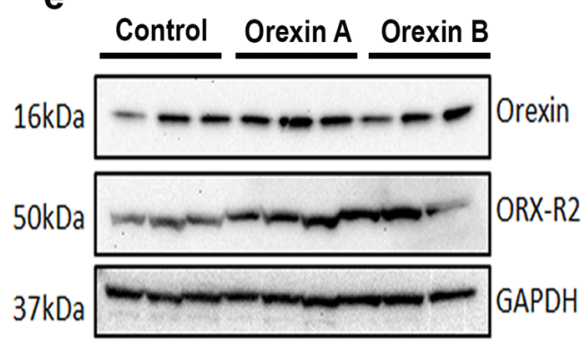

h

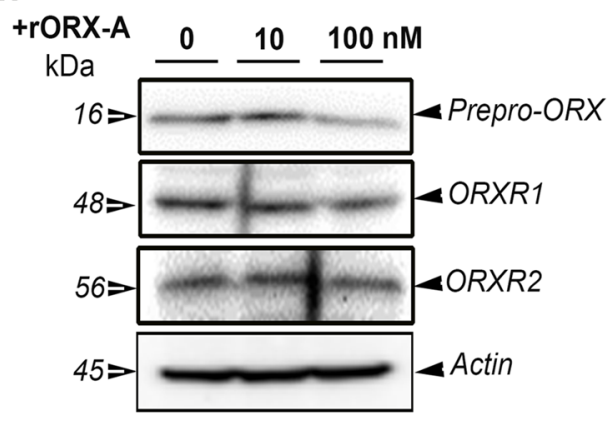

k

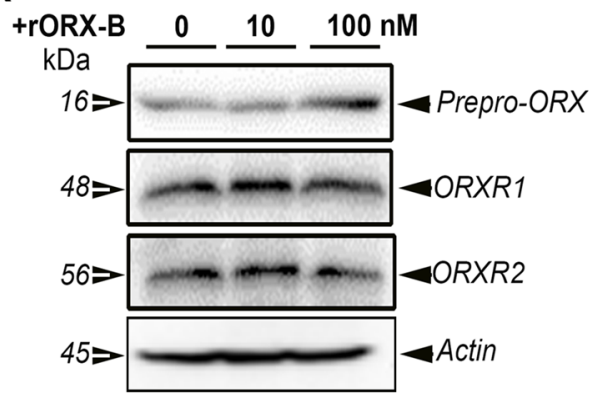

C

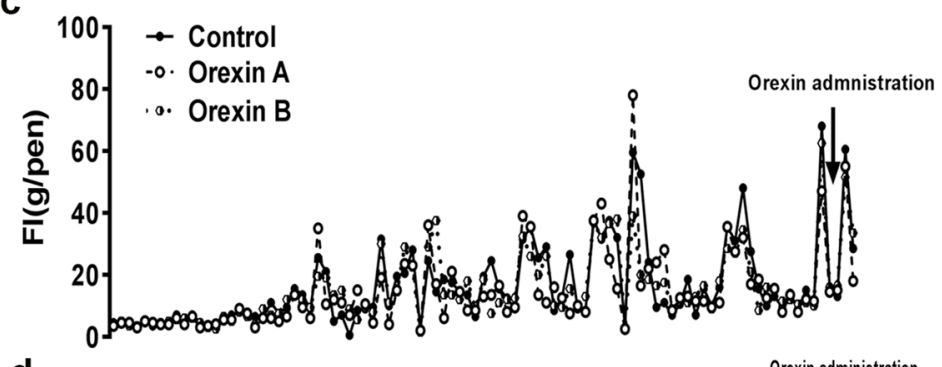

d

- Control

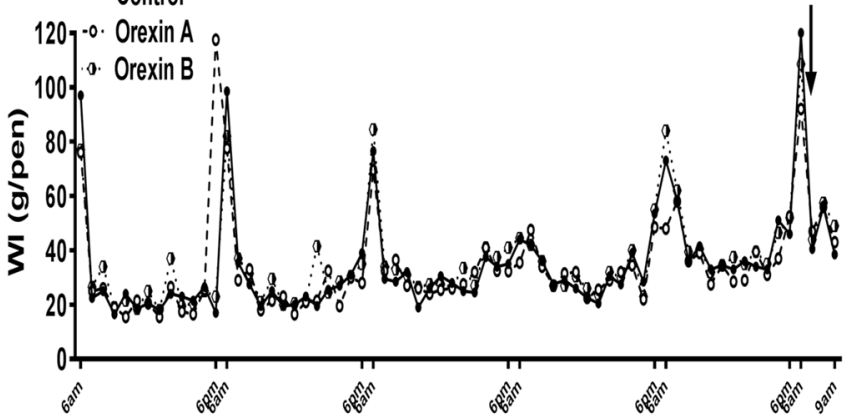

f

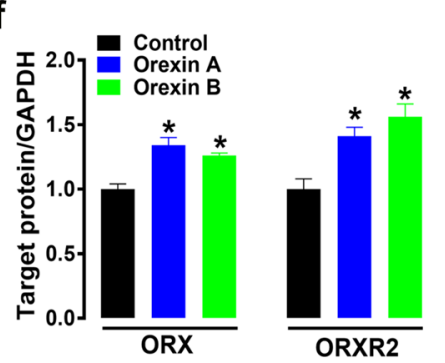

g

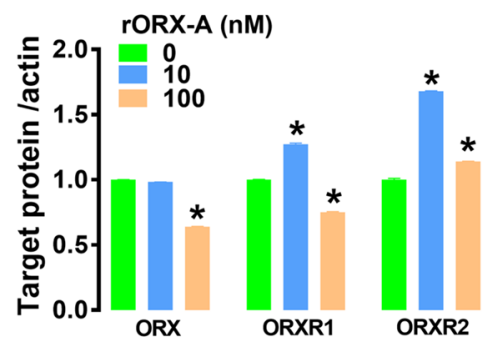

j

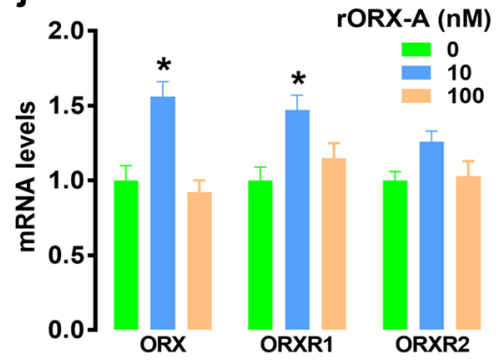

Figure 3. Orexin regulates its own system in chicken liver and LMH cells. Intraperitoneal injection of human recombinant orexins (A and B, $100 \mathrm{ng} / 100 \mathrm{~g} \mathrm{BW}$ ) affects blood orexin mRNA levels (a), BW (b), FI (c), WI (d), and liver expression of orexin and ORXR2 at protein $(\mathbf{e}, \mathbf{f})$ and mRNA levels $(\mathbf{g})$. Orexin treatment affects orexin, ORXR1, and ORXR2 at protein $(\mathbf{h}, \mathbf{i}, \mathbf{k}, \mathbf{l})$ and mRNA levels $(\mathbf{j}, \mathbf{m})$ in LMH cells. mRNA abundances and protein levels were determined by qPCR and Western blot, respectively as described in "Materials and methods". Data are presented as mean \pm SEM $\left(n=12\right.$ birds/group). ${ }^{*}$ Significant difference from controls, $P<0.05$. Western blot figures are representative of 4 replicates. $B W$ body weight, FI feed intake, $W I$ water intake. Full-length blots/gels are presented in Supplementary Fig. S1. 

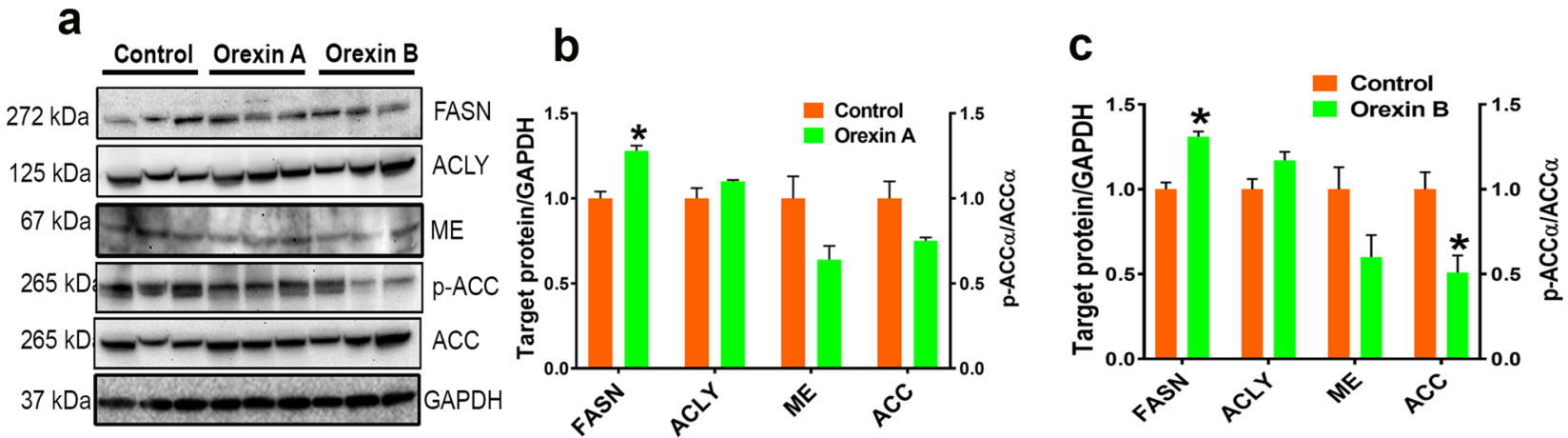

d

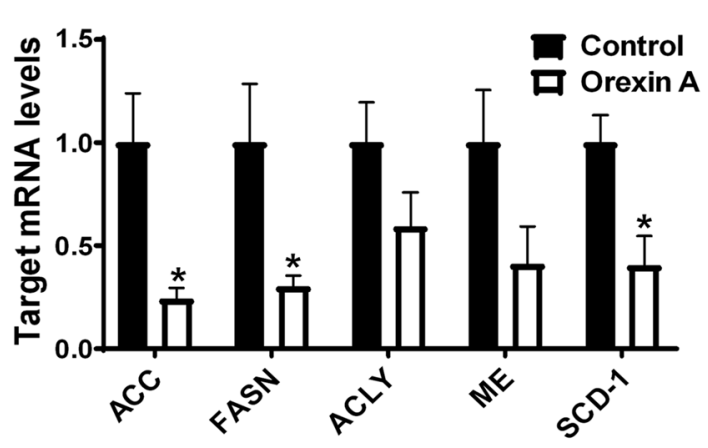

f

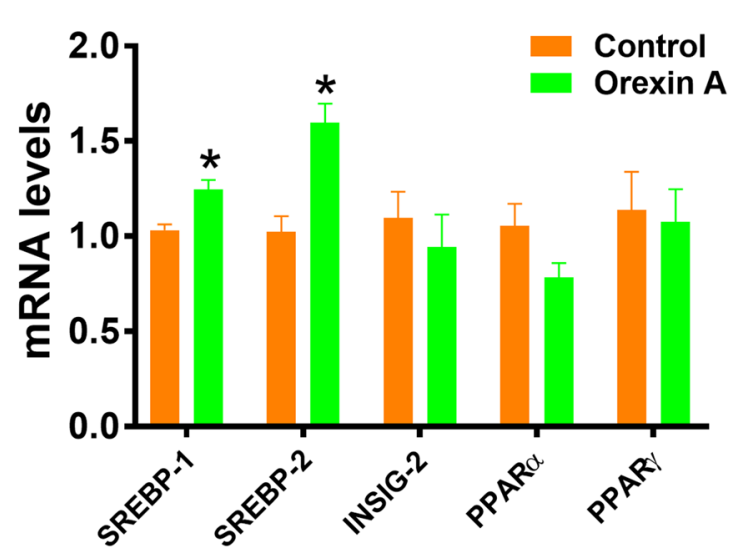

Target TF e

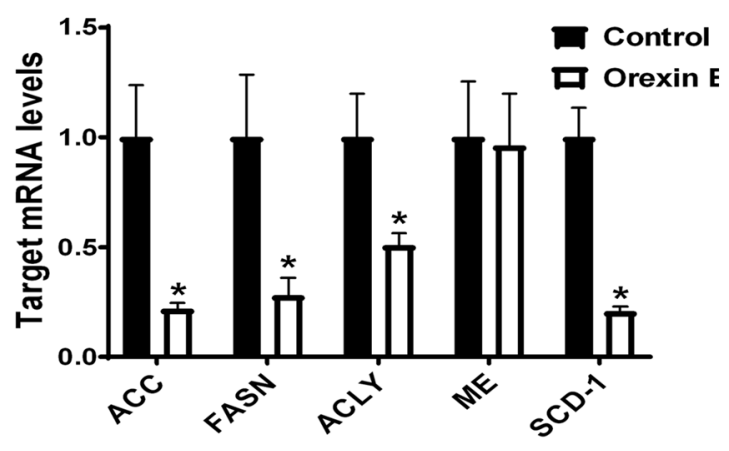

g

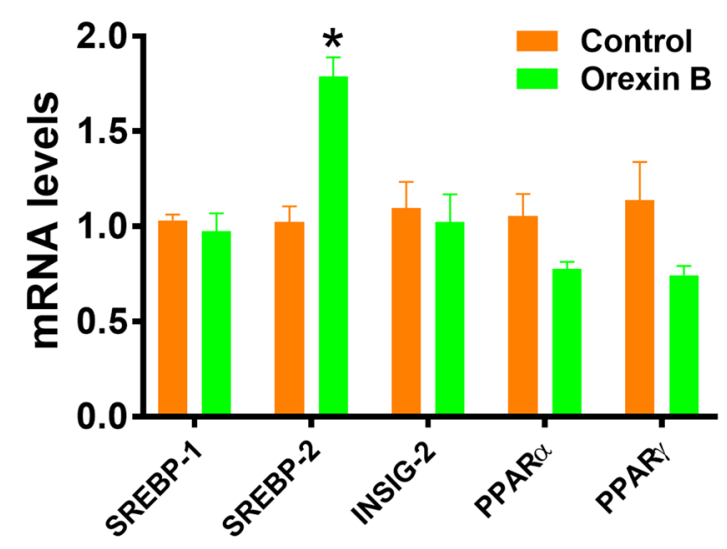

Target TF

Figure 4. Orexin regulates lipogenic program in chicken liver. Intraperitoneal administration of human recombinant orexins affects lipogenic factors (ACC, FASN, ACLY, ME, SCD-1) and their transcription factors. Protein expression $(\mathbf{a}-\mathbf{c})$ was determined by Western blot and gene expression $(\mathbf{d}-\mathbf{g})$ was measured by qPCR. Data are presented as mean \pm SEM $\left(\mathrm{n}=12\right.$ birds/group). ${ }^{\star}$ Indicates significant difference from controls at $P<0.05$. ACC acetyl-CoA carboxylase, $A C L Y$ ATP citrate lyase, FASN fatty acid synthase, INSIG-2 insulininduced gene 2, ME malic enzyme, PPAR peroxisome proliferator-activated receptor, $S C D$ - 1 stearoyl-CoA desaturase-1, $S R E B P-1 / 2$ sterol regulatory element-binding protein 1/2. Full-length blots/gels are presented in Supplementary Fig. S1.

\section{Discussion}

Orexin A and B neuropeptides have attracted much recent attention due to their pleiotropic physiological functions. They were initially characterized in the brain and first emerged as appetite inducers and regulators of 
a

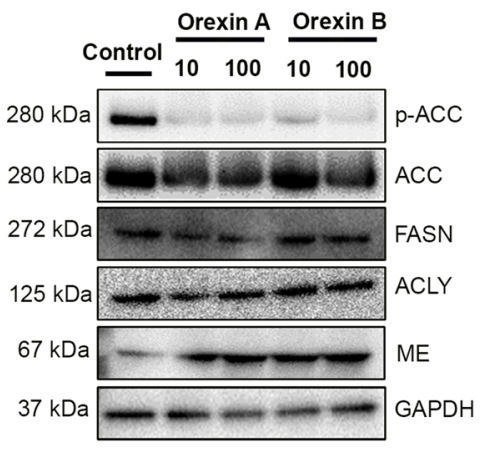

b

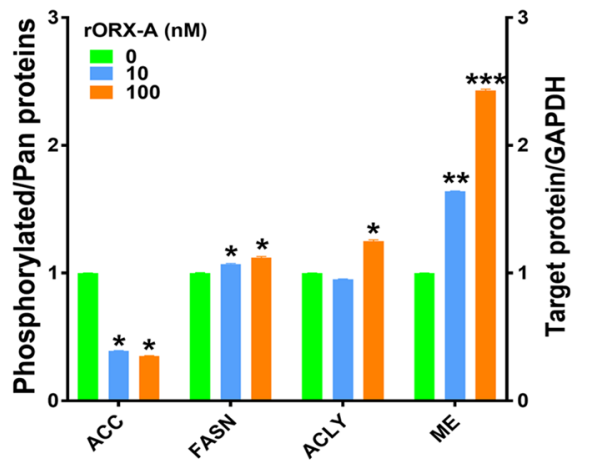

d Control
C

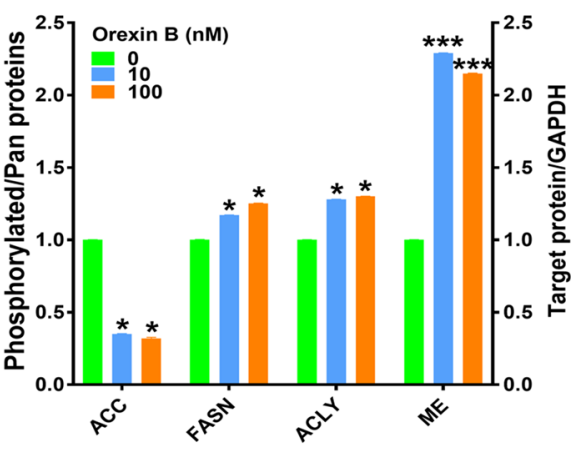

e

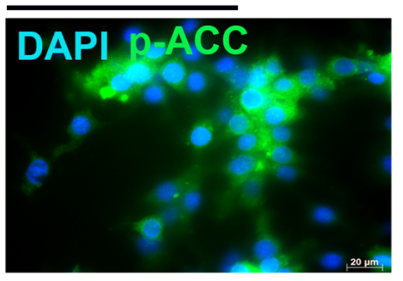

+ORX-B
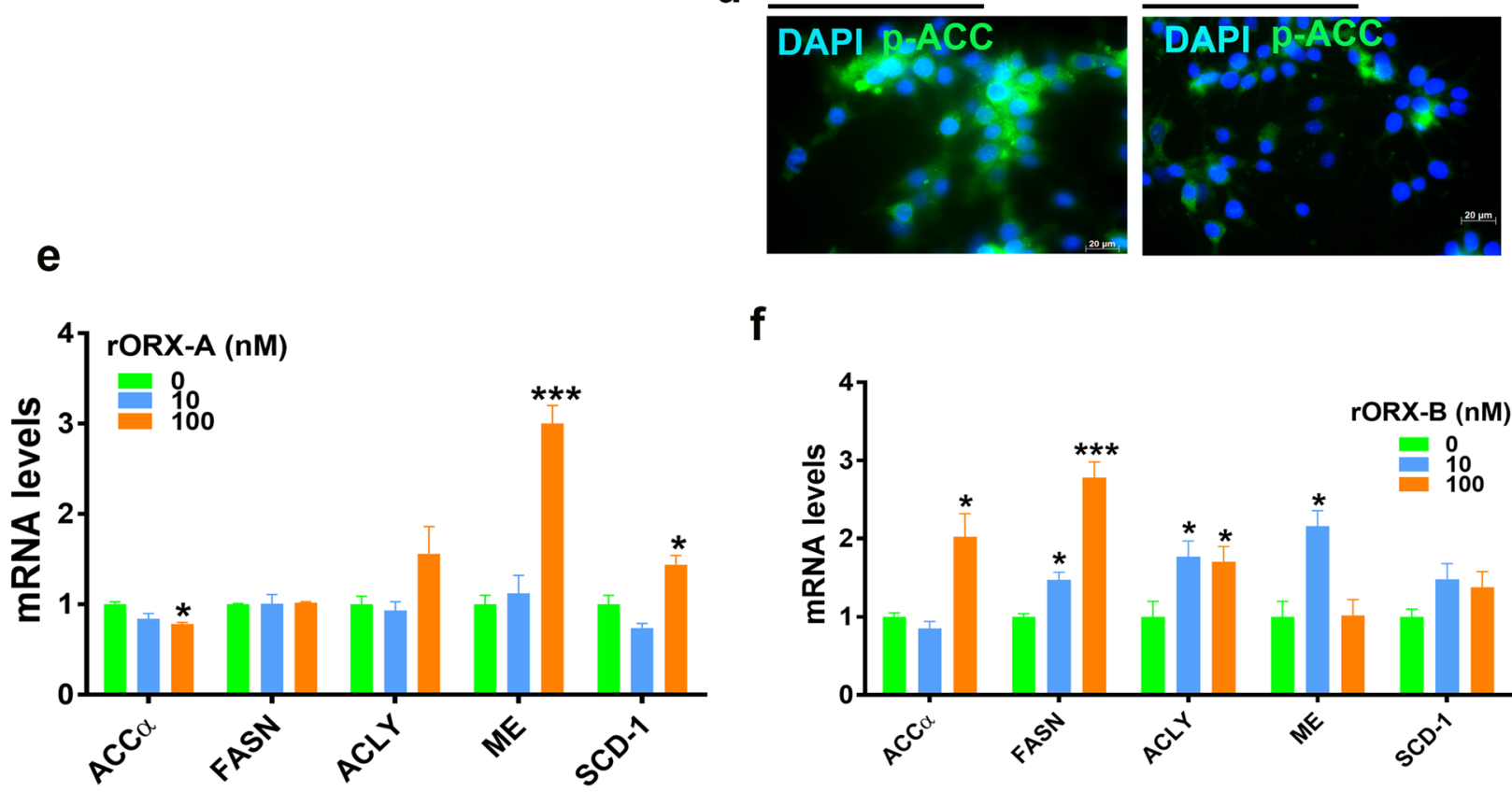

f

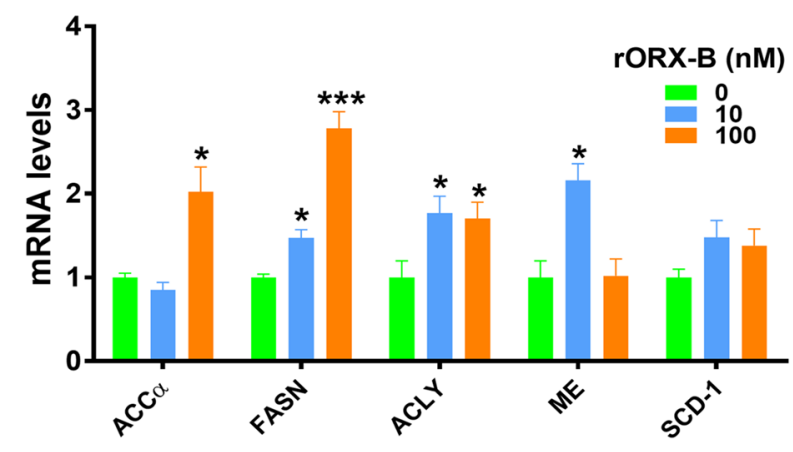

g
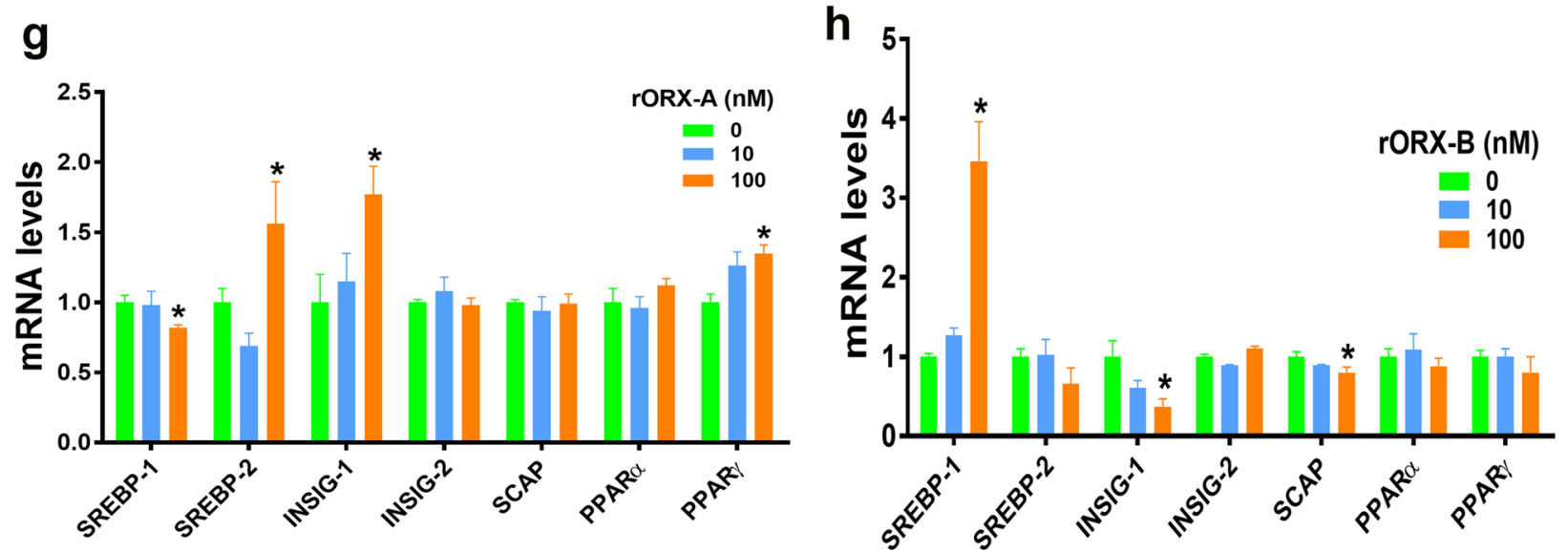

Figure 5. Orexin regulates lipogenic program in $\mathrm{LMH}$ cell line. Orexin treatments affects lipogenic factors (ACC, FASN, ACLY, ME, SCD-1) and their transcription factors. Protein expression $(\mathbf{a}-\mathbf{c})$ was determined by Western blot and gene expression (e-h) was measured by qPCR. Phosphorylated levels of ACC was also assessed by immunofluorescence staining $(\mathbf{d})$. Data are presented as mean \pm SEM $\left(n=6\right.$ /group). ${ }^{*}$ Indicates significant difference from controls at $P<0.05$. ACC acetyl-CoA carboxylase, ACLY ATP citrate lyase, FASN fatty acid synthase, INSIG-2 insulin-induced gene 2, ME malic enzyme, $P P A R$ peroxisome proliferator-activated receptor, $S C D-1$ stearoyl-CoA desaturase-1, SREBP-1/2 sterol regulatory element-binding protein 1/2. Fulllength blots/gels are presented in Supplementary Fig. S1. 


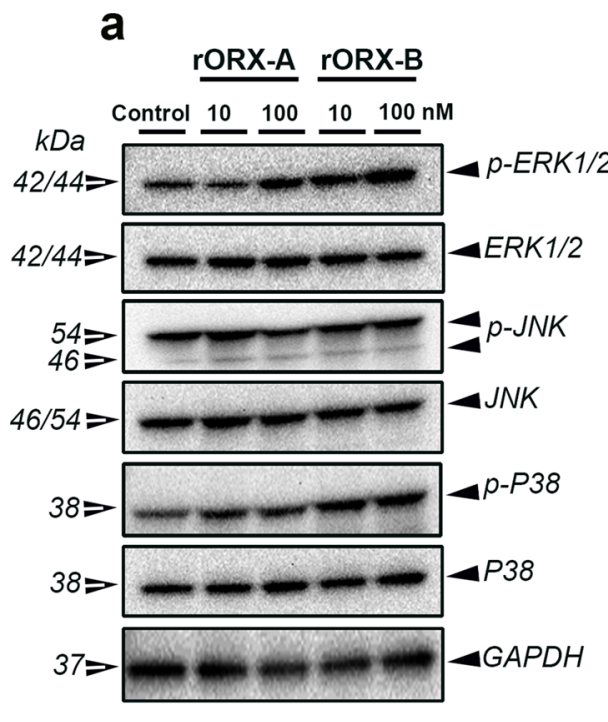

b

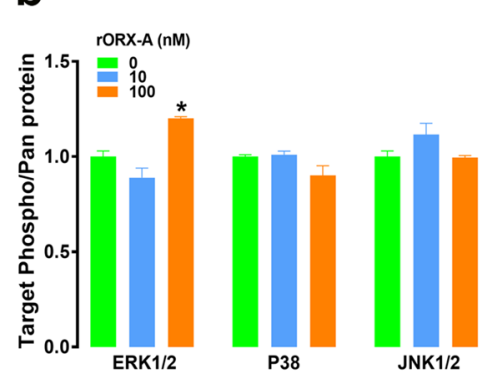

d

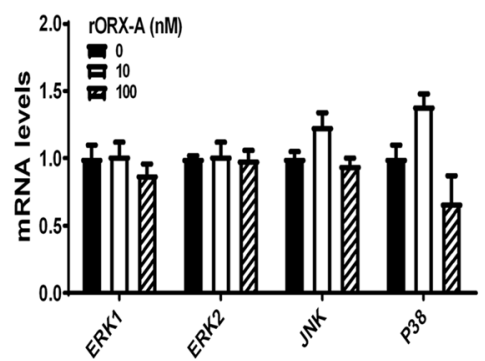

C

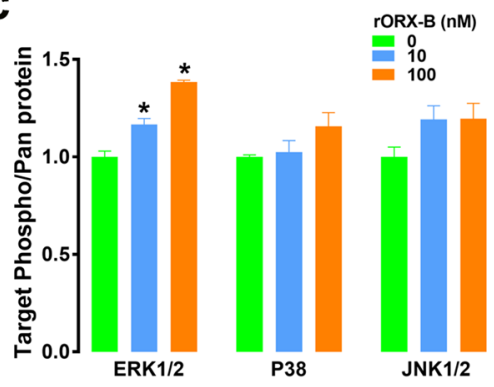

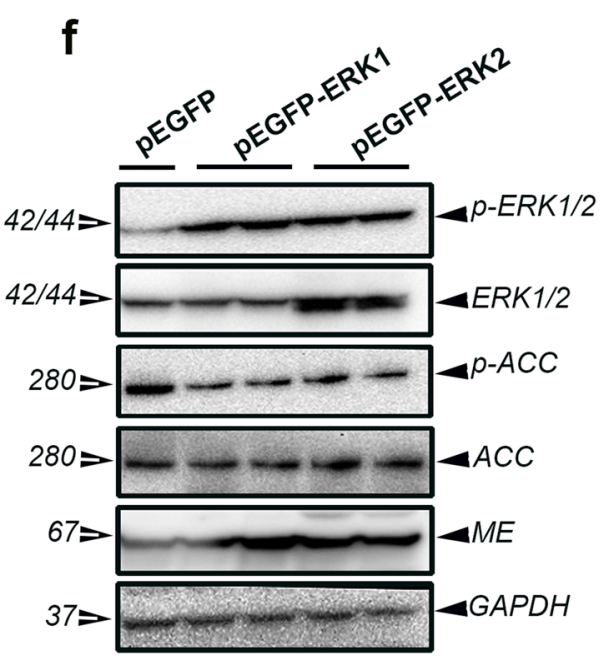

g
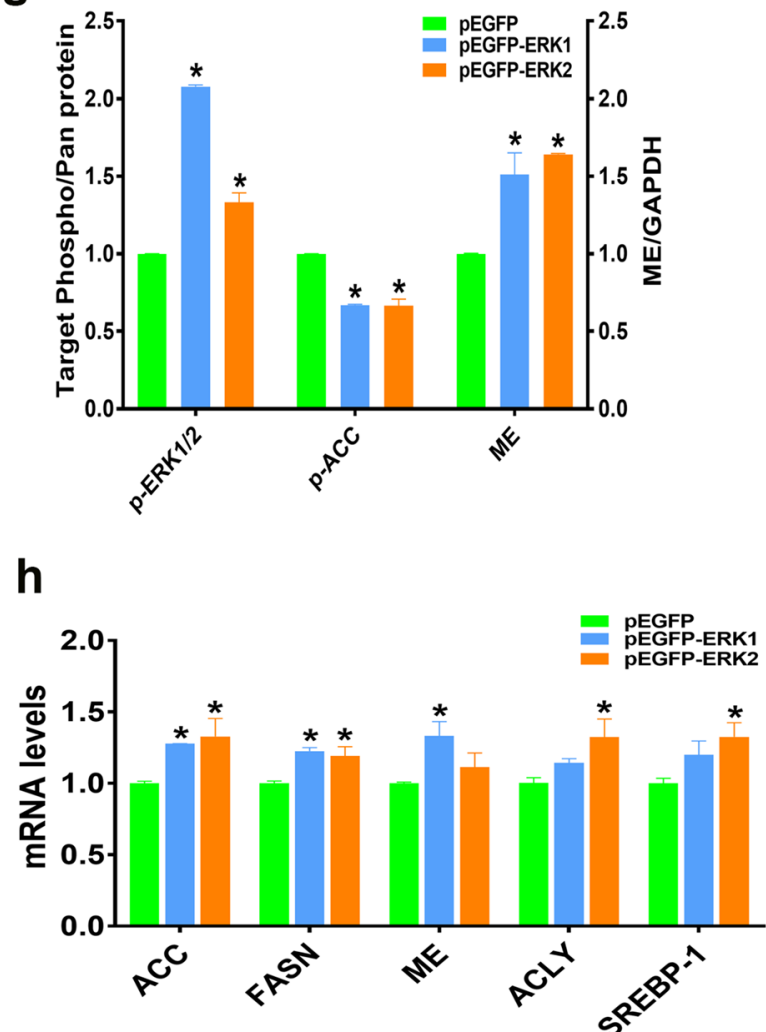

Figure 6. Orexin activates ERK1/2 signaling cascade in LMH cells. Orexin administration increased p-ERK1/2 ${ }^{\text {Thr202/Tyr204 }}$ levels, but not that of p-P38 ${ }^{\text {Thr180/Tyr182 }}$ or p-JNK1/2 ${ }^{\text {Thr183/Tyr185 }}$ in LMH cells as demonstrated by Western blot analyses $(\mathbf{a}-\mathbf{c})$. The relative expression of MAPK genes was measured by qPCR $(\mathbf{d}, \mathbf{e})$.

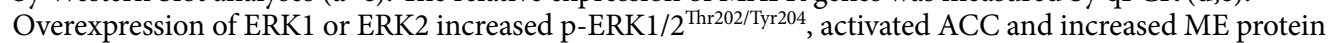
levels $(\mathbf{f}, \mathbf{g})$ as well as the mRNA abundances of ACC, FASN, ME, ACLY and SREBP-1 (h). Data are presented as mean $\pm \operatorname{SEM}(\mathrm{n}=6 /$ group$) .{ }^{*}$ Indicates significant difference from controls at $P<0.05$. ACC acetyl-CoA carboxylase, ACLY ATP citrate lyase, ERK extracellular signal-regulated kinase, FASN fatty acid synthase, $J N K$ c-Jun N-terminal kinase, $M E$ malic enzyme, P38 P38 mitogen-activated protein kinase, SREBP-1 sterol regulatory element-binding protein 1. Full-length blots/gels are presented in Supplementary Fig. S1. 


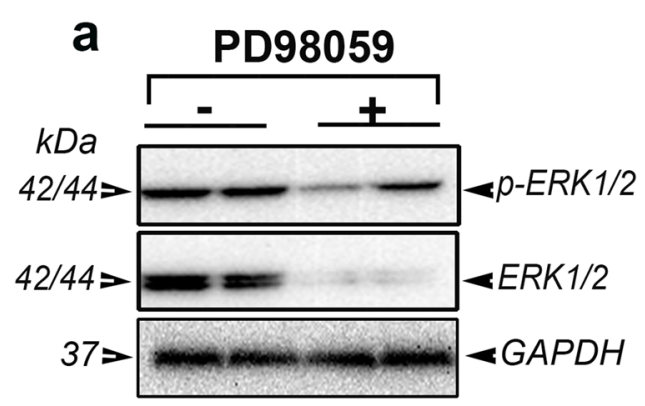

b

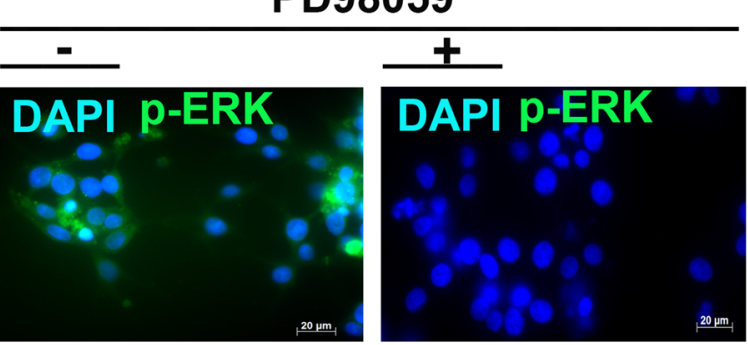

C

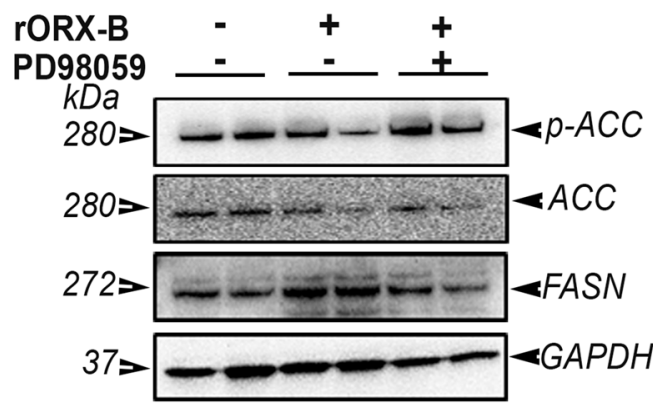

d
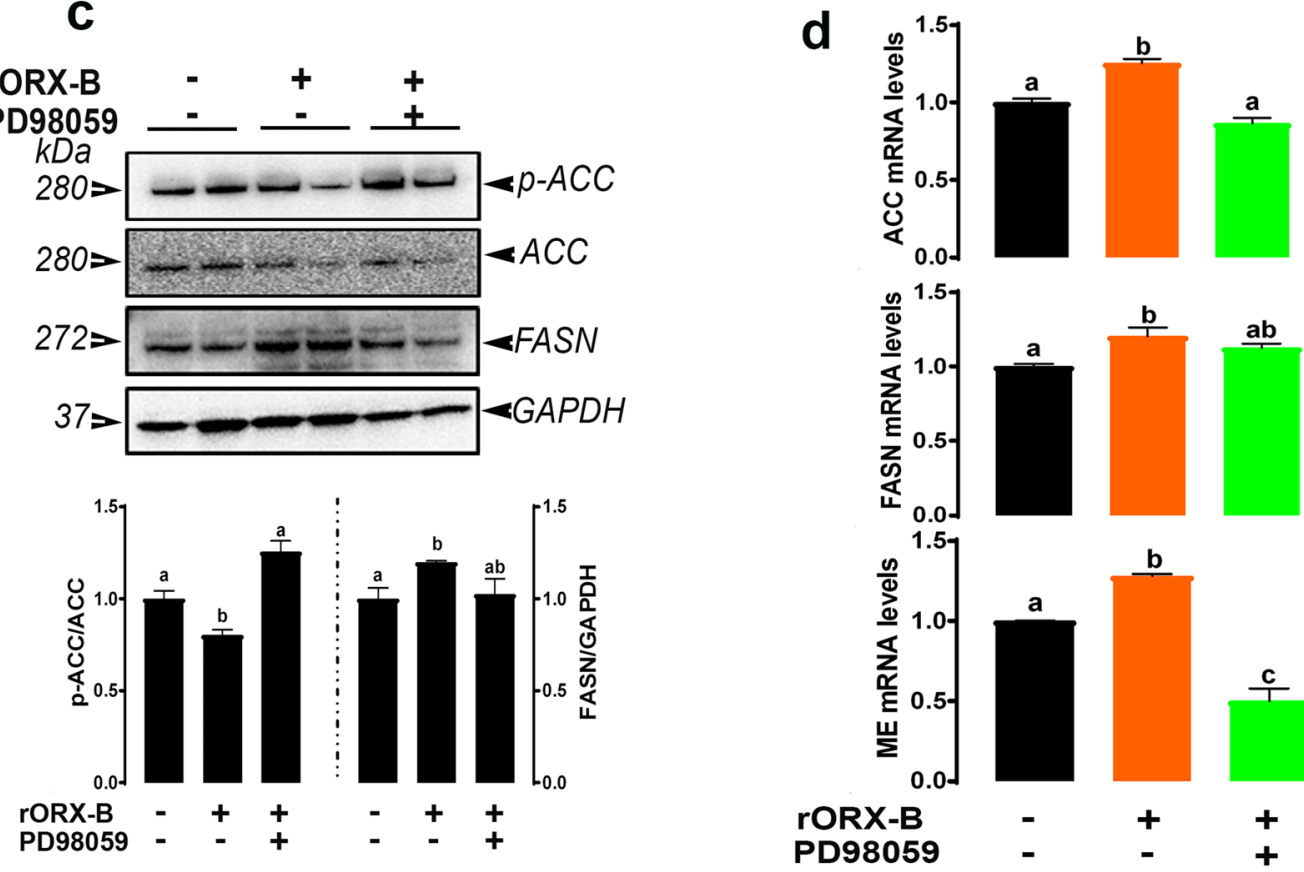

e
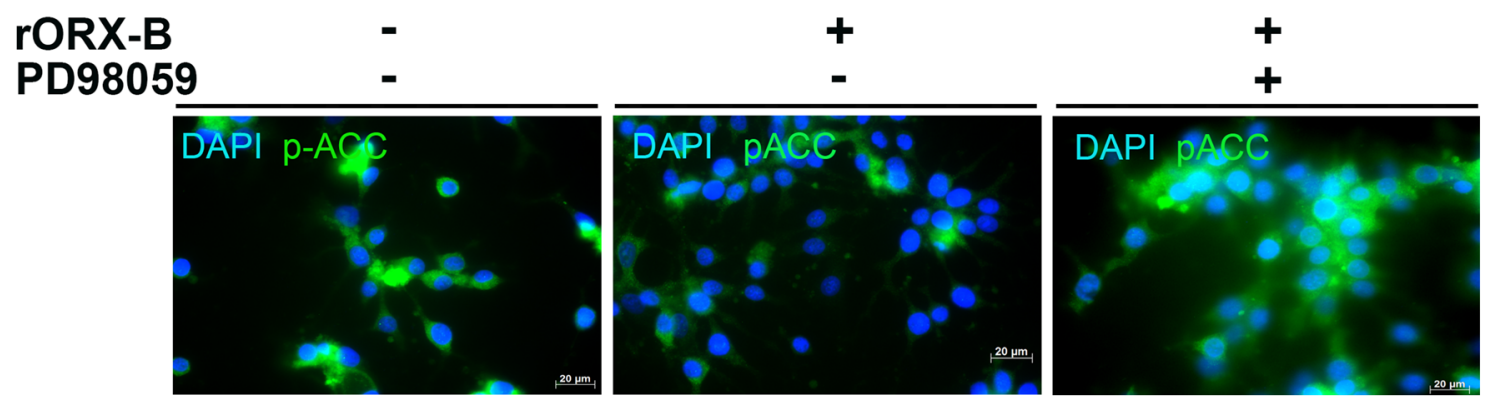

Figure 7. Blockade ERK1/2 activation prevents orexin-induced lipogenesis in LMH cells. Pharmacological inhibition of ERK1/2 using PD98059, as confirmed by Western blot (a) and immunofluorescence (b), blocked the effect of orexin on the expression lipgenic factors (c-e). Data are presented as mean \pm SEM ( $n=6 /$ group). ${ }^{\star}$ Indicates significant difference from the untreated group (control) at $P<0.05$. ACC acetyl-CoA carboxylase, ERK extracellular signal-regulated kinase, FASN fatty acid synthase. Full-length blots/gels are presented in Supplementary Fig. S1.

feeding behaviors and energy homeostasis in mammals ${ }^{4}$. Numerous studies showed that orexins and their two known G-protein-coupled receptors are expressed in many peripheral tissues and play crucial roles in sleep and wakefulness cycle ${ }^{6-8}$, energy metabolism ${ }^{9,10}$, stress response ${ }^{13,14}$, and glucose and lipid metabolism ${ }^{23,29}$ in mammalian species. Although orexins were found to be expressed not only in the hypothalamus of chickens (nonmammalian species $)^{17}$, but also in gastrointestinal $\operatorname{tract}^{18}$, reproductive organs ${ }^{19}$, and muscle ${ }^{20}$, their functions are still not well defined. The objective of the current study was to investigate the potential role of the orexin system in avian hepatic lipid metabolism. As in humans, chicken liver is the primary site of de novo fatty acid synthesis ${ }^{21}$. 


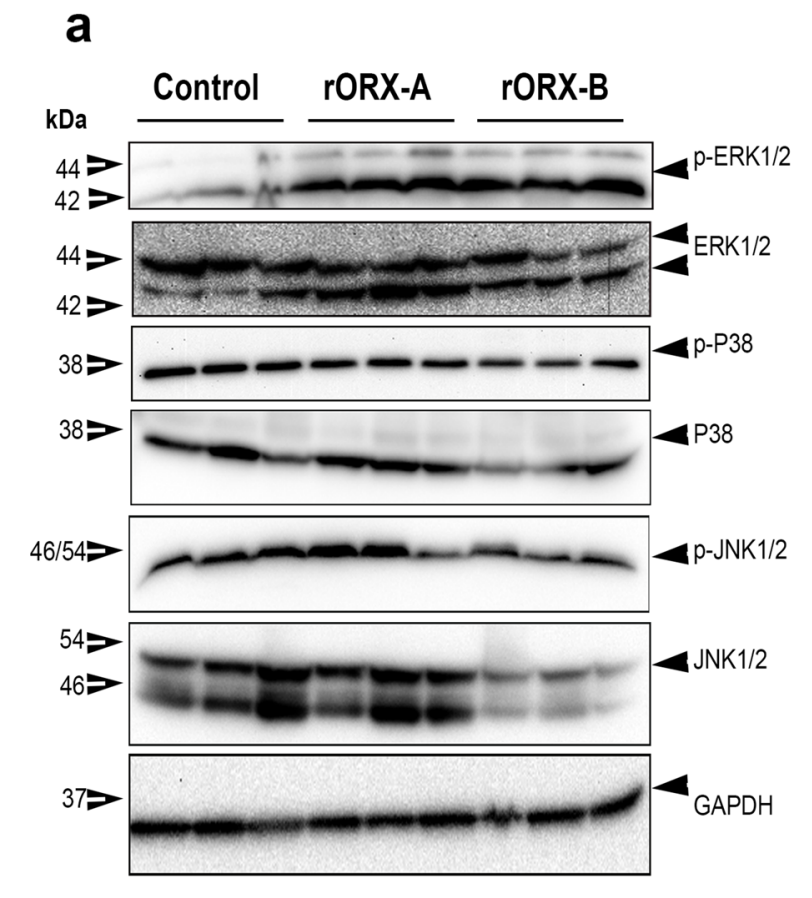

b
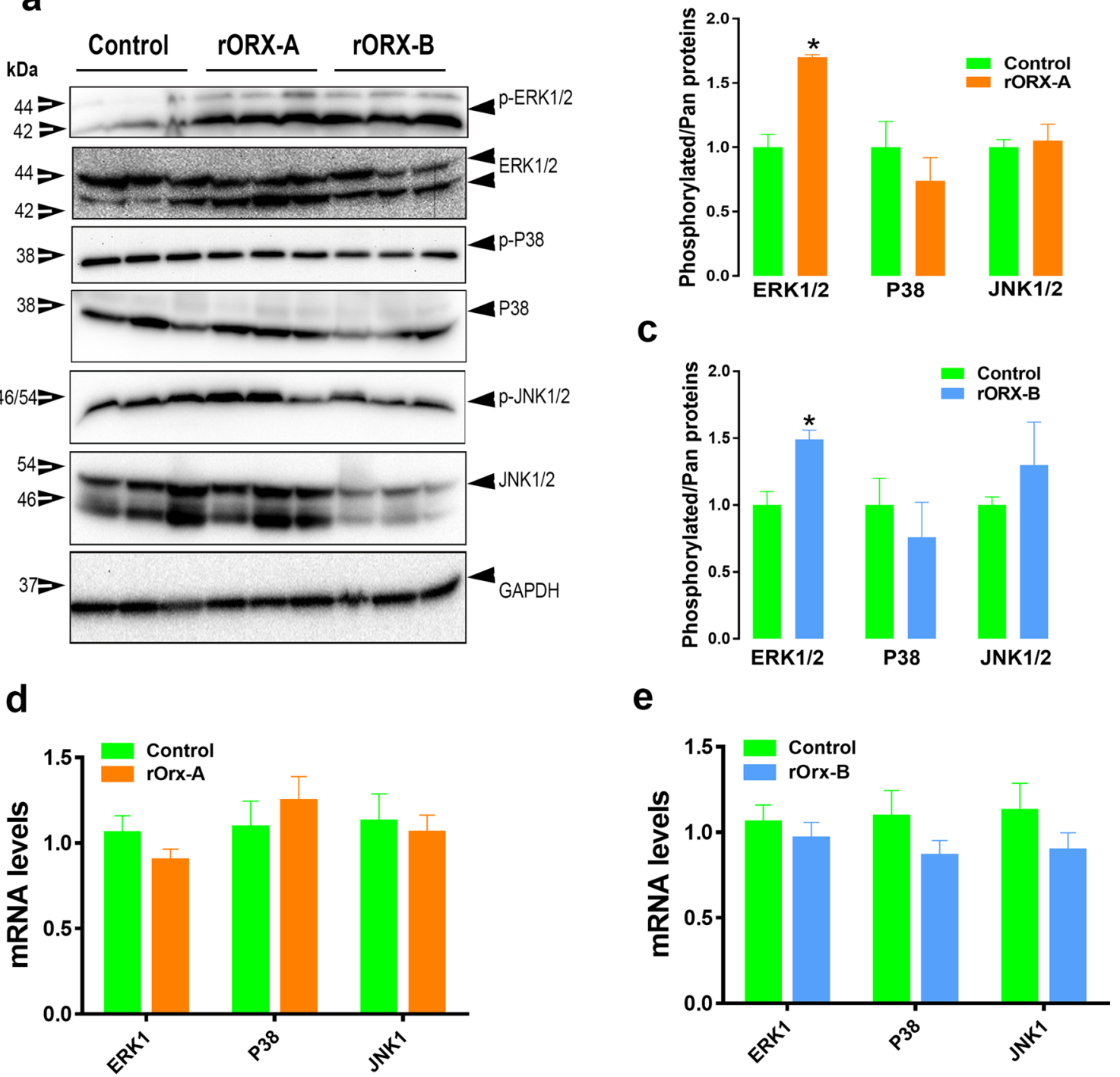

Figure 8. Orexins activate ERK1/2 signaling cascade in chicken liver. Intraperitoneal administration of recombinant orexins increased p-ERK1/2 ${ }^{\text {Thr202/Tyr204 }}$ levels, but not that of p-P38 ${ }^{\text {Thr180/Tyr182 }}$ or p-JNK1/2 ${ }^{\text {Thr183/ }}$ ${ }^{T y r} 185$ in chicken liver as demonstrated by Western blot analyses $(\mathbf{a}-\mathbf{c})$. The relative expression of MAPK genes was measured by qPCR (d,e). Data are presented as mean \pm SEM ( $\mathrm{n}=12$ birds/group). ${ }^{\star}$ Indicates significant difference from controls at $P<0.05$; ERK extracellular signal-regulated kinase, JNK c-Jun N-terminal kinase, $P 38$ P38 mitogen-activated. Full-length blots/gels are presented in Supplementary Fig. S1.

Additionally, commercial broiler (meat-type) chicken are often hyperphagic and prone to fat accumulation ${ }^{30}$. Since obesity is a worldwide epidemic, these peculiarities make the chicken a high relevant animal model for further understanding hepatic lipid metabolism in both poultry and humans.

By using different techniques (qPCR, immunoblot, and immunofluorescence staining), we first demonstrated that orexin and its related receptors ORXR1 and ORXR2 are expressed in chicken liver and LMH cells. Cell fractionation showed that orexin system is mainly localized in the cytoplasm. Further subcellular analyses by double-labeling immunofluorescence indicated that orexin is localized in ER, Golgi apparatus, and in the lysosome. Although the intracellular distribution of orexin system has not been reported previously, several studies showed that orexin system couples to $\mathrm{Ca}^{2+}$ influx and release $\mathrm{e}^{31-33}$. As the ER plays a key role in calcium homeostasis and storage ${ }^{34}$, together these studies suggested that orexin might be localized in the ER which is supported by COMPARTMENTS, the subcellular localization database (https://compartments.jensenlab.org). Additionally, orexin contains an ER signal sequence ${ }^{35}$. The presence of orexin in ER and Golgi as well as in the culture media indicated that orexin is secreted from chicken hepatocytes. This is confirmed by brefeldin A treatment, the potent inhibitor of secretion via disruption and disassembly of the Golgi leading to the accumulation of orexin in the cell lysate ${ }^{36}$. The localization of orexin in the lysosome suggested that orexin might be involved in autophagosome-lysosome fusion process and autophagy machinery $y^{37,38}$. 
Although both recombinant orexins upregulated the hepatic orexin system in vivo, rORX-A and rORX-B had differential effects on ORX protein expression in LMH cells. These differences might be due to structural differences between ORX-A and -B (ORX-A contains two disulfide bonds as compared to one in ORX-B) and/ or binding affinity of the orexins to their receptors ${ }^{39}$. In fact, mammalian ORXR1 preferentially binds ORX-A, while ORXR2 has similar affinity for both ORX-A and - $\mathrm{B}^{1}$. Additionally, heterodimerization with other G-protein coupled receptors is known to affect orexin receptor activation ${ }^{40}$. Determination of the crystal structure of the chicken orexin receptors as well as their ligand-binding properties may help to further elucidate the differential actions of orexin-A and $-\mathrm{B}^{41}$. Similarly, changes in mRNA and protein expression of orexin system are not concomitant. For instance, orexin administration upregulated ORX and ORXR2 protein levels without affecting their mRNA abundances. This lack of correlation between mRNA and protein levels is not surprising ${ }^{42}$ and it $^{2}$ might be explained by the involvement of varied post-transcriptional and post-translational mechanisms and/ or protein and RNA half- lives ${ }^{43}$. The hepatic expression and secretion of orexin system as well as its ability to regulate its own system suggested that orexin might have autocrine, paracrine, and/or endocrine function ${ }^{44}$.

Though originally identified as a regulator of feeding behavior in mammals, orexin does not appear to have this effect in chicken in our study and that of others ${ }^{45}$, leading to questions regarding its role in avian physiology. Here we showed for the first time that orexin administration in vivo increased hepatic FASN protein levels, which is a rate-limiting enzyme of fatty acid synthesis via catalyzing the synthesis of palmitate from acetyl-CoA and malonyl-CoA into long-chain saturated fatty acids ${ }^{46}$. In support of the in vivo data, orexin treatments also induced the expression of key lipogenic proteins including FASN, ACLY and ME in LMH cells. Reduction of its phosphorylated levels indicated the activation of $\mathrm{ACCa}$, which constitutes a key control point in the synthesis of long-chain fatty acids through catalyzing the carboxylation of acetyl-CoA in the generation of malonyl-CoA ${ }^{47}$. In line with our results, previous studies have shown that orexin stimulated lipid accumulation in primary rat adipocytes and 3T3-L1 cells ${ }^{23}$. Pruszynska-Oszmalek and co-workers reported that orexin stimulated proliferation and differentiation of porcine preadipocytes and regulated lipid metabolism ${ }^{29,48}$. It is noteworthy to mention that, as for orexin system and for the same reasons mentioned above, mRNA and protein levels of lipogenic factors were not correlated. Interestingly, in vivo administration of rORX-A up-regulated both hepatic SREBP-1 and SREBP-2, however rORX-B induced only SREBP-2 gene expression. In LMH cells, however, rORX-A downregulated SREBP-1 and up-regulated SREBP-2 and INSIG-1 gene expression, and rORX-B increased SREBP-1 and decreased $I N S I G-1$ and SCAP mRNA abundances. Although the mechanisms contributing to the differential regulation of transcription factors SREBP-1/2 between rORX-A and rORX-B on one hand, and between in vivo and in vitro studies on the other hands, are not known, it is possible that other transcription factors might be involved. For instance, the up-regulation of PPAR $\gamma$ in LMH cells following rORX-A treatment might compensate for the down-regulation of SREBP-1. It has been shown that PPAR $\gamma$ is one of the downstream mediators of orexin's action ${ }^{23}$. The consistent increase in SREBP-2 mRNA levels following rORX-A administration in both chicken liver and LMH cells indicated that at least rORX-A regulate cholesterol metabolism ${ }^{49}$.

Orexin has been shown to activate various signaling pathways ${ }^{4,50}$ and lipogenic enzymes have been reported to be regulated by diverse mechanisms ${ }^{51}$. In attempt to define the downstream mediators of orexin action on avian hepatic lipogenesis, we sought to determine the involvement of MAPK cascades. Orexin administration led to ERK1/2 phosphorylation at Thr202/Tyr204 residues, but not that of P38 and JNK1/2 in both chicken liver and LMH cells. Overexpression and activation of ERK1 or ERK2 up-regulated the expression of lipogenic genes (ACCa, FASN, ME, and ACLY), and their transcription factor SREBP-1, increased ME protein levels, and activated ACCa as demonstrated by its dephosphorylation. Pharmacological inhibition of ERK1/2 activation by PD98059 prevents orexin-induced lipogenesis. Orexin has been shown to activate ERK1/2 in multiple cell types and species, including CHO cells ${ }^{52}$, SGC-7901 gastric cancer cells ${ }^{53}, \mathrm{H} 295 \mathrm{R}$ adrenocortical cells ${ }^{54}$, neuro2a cells ${ }^{55}$, and 3T3-L1 cells ${ }^{56}$. ERK1/2 signaling pathway is involved in a wide variety of cellular processes. Roth and co-workers ${ }^{57}$ have shown that ERK1/2 regulated the key lipogenic transcription factor SREBP-1 and several lines of evidence have implied a link between ERK1/2 and lipid metabolism in mammals ${ }^{58-61}$. Although further in-depth investigations are needed to delineate the mechanisms by which orexin activate ERK1/2 in chicken hepatocytes, we speculate that Gq/Gi, PLC, and PKC are potentially involved ${ }^{54,62}$.

In conclusion, this is the first report evidencing the hepatic expression of orexin system in avian (nonmammalian) species and its role in lipogenesis via ERK1/2 pathway. This is significant because it identified a new molecular signature that could open new vistas for mechanistic understanding of avian hepatic lipid metabolism.

Received: 5 February 2020; Accepted: 22 October 2020

Published online: 05 November 2020

\section{References}

1. Sakurai, T. et al. Orexins and orexin receptors: a family of hypothalamic neuropeptides and G protein-coupled receptors that regulate feeding behavior. Cell 92, 573-585 (1998).

2. de Lecea, L. et al. The hypocretins: hypothalamus-specific peptides with neuroexcitatory activity. Proc. Natl. Acad. Sci. 95, 322-327 (1998).

3. Heinonen, M. V., Purhonen, A. K., Makela, K. A. \& Herzig, K. H. Functions of orexins in peripheral tissues. Acta. Physiol. 192, 471-485 (2008).

4. Li, J., Hu, Z. \& de Lecea, L. The hypocretins/orexins: integrators of multiple physiological Functions. Br. J. Pharmacol. 171, 332-350. https://doi.org/10.1111/bph.12415 (2014).

5. Sakurai, T. Orexins and orexin receptors: implication in feeding behavior. Regul. Peptides. 85, 25-30 (1999).

6. Chemelli, R. et al. Narcolepsy in orexin knockout mice: molecular genetics of sleep regulation. Cell 98, 437-451 (1999).

7. Lin, L. et al. The sleep disorder canine narcolepsy is caused by a mutation in the hypocretin (orexin) receptor 2 gene. Cell 98 , 365-376 (1999). 
8. Piper, D., Upton, N., Smith, M. \& Hunter, A. The novel brain neuropeptide, orexin-A, modulates the sleep-wake cycle of rats. Eur. J. Neurosci. 12, 726-730 (2000).

9. Tsuneki, H., Wada, T. \& Sasaoka, T. Role of orexin in the central regulation of glucose and energy homeostasis. Endocr. J. 59, 365-374 (2012).

10. Tsuneki, H., Wada, T. \& Sasaoka, T. Role of orexin in the regulation of glucose homeostasis. Acta. Physiol. 198, 335-348. https:// doi.org/10.1111/j.1748-1716.2009.02008 (2010).

11. Shirasaka, T., Nakazato, M., Matsukura, S., Takasaki, M. \& Kannan, H. Sympathetic and cardiovascular actions of orexins in conscious rats. Am. J. Physiol. 277, R1780-R1785 (1999).

12. Samson, W. K., Gosnell, B., Chang, J. K., Resch, Z. T. \& Murphy, T. C. Cardiovascular regulatory actions of the hypocretins in brain. Brain Res. 831, 248-253 (1999).

13. Ida, T. et al. Possible involvement of orexin in the stress reaction in rats. Biochem. Biophys. Res. Commun. 270, 318-323. https:// doi.org/10.1006/bbrc.2000.2412 (2000).

14. Winsky-Sommerer, R. et al. Interaction between the corticotropin-releasing factor system and hypocretins (orexins): a novel circuit mediating stress response. J. Neurosci. 24, 11439-11448. https://doi.org/10.1523/JNEUROSCI.3459-04.2004 (2004).

15. Digby, J. et al. Orexin receptor expression in human adipose tissue: effects of orexin-A and orexin-B. J. Endocrinol. 191, 129-136 (2006).

16. Shen, Y. et al. Effects of orexin A on GLUT4 expression and lipid content via MAPK signaling in 3T3-L1 adipocytes. J. Steroid Biochem. Mol. Biol. 138, 376-383 (2013).

17. Ohkubo, T., Boswell, T. \& Lumineau, S. Molecular cloning of chicken prepro-orexin cDNA and preferential expression in the chicken hypothalamus. Biochem. Biophys. Acta. 1577, 476-480 (2002).

18. Aracmaone, N. et al. Orexin and orexin receptor like peptides in the gastrointestinal tract of Gallus domesticus: an immunohistochemical survey on presence and distribution. Res. Vet. Sci. 96, 234-240 (2014).

19. Ohkubo, T., Tsukada, A. \& Shamoto, K. cDNA cloning of chicken orexin receptor and tissue distribution: sexually dimorphic expression in chicken gonads. J. Mol. Endocrinol. 31, 499-508 (2003).

20. Lassiter, K. et al. Orexin system is expressed in avian muscle cells and regulates mitochondrial dynamics. Am. J. Physiol. Regul. Integr. Comp. Physiol. 308, R173-R187. https://doi.org/10.1152/ajpregu.00394.2014 (2015).

21. Leveille, G. A., Romsos, D. R., Yeh, Y., \& O'Hea, E. K. Lipid biosynthesis in the chick. A consideration of site of synthesis, influence of diet and possible regulatory mechanisms. Poult. Sci. 54, 1075-1093 (1975).

22. Lee, J. et al. Establishment of an immortal chicken embryo liver-derived cell line. Poult. Sci. 92, 1604-1612 (2013).

23. Skrzypski, M. et al. Orexin A stimulates glucose uptake, lipid accumulatio and adiponectin secretion from 3T3-L1 adipocytes and isolated rat primary hepatocytes. Diabetologia 54, 1841-1852 (2011).

24. Butterick, T. A., Nixon, J. P., Billington, C. J. \& Kotz, C. M. Orexin A decreases lipid peroxidation and apoptosis in a novel hypothalamic cell model. Neurosci. Lett. 524, 30-34 (2012).

25. Dridi, S. et al. ERK1/2 activation is a therapeutic target in age-related macular degeneration. Proc. Nat. Acad. Sci. 109, 13781-13786. https://doi.org/10.1073/pnas.1206494109 (2012).

26. Dhamad, A. E., et al. $75-\mathrm{kD}$ glucose-regulated protein (GRP75) is a novel molecular signature for heat stress response in avian species. Am. J. Physiol.-Cell Physiol. 318, C289-C303. https://doi.org/https://doi.org/10.1152/ajpcell.00334.2019 (2020).

27. Greene, E. et al. Double-stranded RNA Is a novel molecular target in osteomyelitis pathogenesis: a translational avian model for human bacterial chondronecrosis with osteomyelitis. Am. J. Pathol. 189, 2077-2089. https://doi.org/10.1016/j.ajpath.2019.06.013 (2019).

28. Schmittgen, T. D. \& Livak, K. J. Analyzing real-time PCR data by the comparative CT method. Nat. Protoc. 3, 1101-1108 (2008).

29. Pruszynska-Oszmalek, E. et al. Orexin A but not orexin B regulates lipid metabolism and leptin secretion in isolated porcine adipocytes. Dom. Anim. Endocrinol. 63, 59-68. https://doi.org/10.1016/j.domaniend.2017.12.003 (2018).

30. Hood, R. The cellular basis for growth of the abdominal fat pad in broiler-type chickens. Poult. Sci. 61, 117-121 (1982).

31. Magga, J. et al. Agonist potency differentiates G protein activation and Ca2+ signalling by the orexin receptor type 1. Biochem. Pharmacol. 71, 827-836 (2006).

32. Tang, J. et al. The signalling profile of recombinant human orexin-2 receptor. Cell. Signal. 9, 1651-1661 (2008).

33. Peltonen, H. M., Åkerman, K. E. O. \& Bart, G. A role for PKD1 and PKD3 activation in modulation of calcium oscillations induced by orexin receptor 1 stimulation. BBA-Mol. Cell Res. 1803, 1206-1212 (2010).

34. Koch, G. L. E. The endoplasmic reticulum and calcium storage. BioEssays 12, 527-531 (1990).

35. Wang, C. et al. The orexin/receptor system: molecular mechanism and therapeutic potential for neurological diseases. Front. Mol. Neurosci. 11, 220. https://doi.org/10.3389/fnmol.2018.00220 (2018).

36. Chardin, P. \& McCormick, F. Brefeldin A: the advantage of being uncompetitive. Cell 97, 153-155 (1999).

37. An, H., Cho, M.-H., Kim, D.-H., Chung, S. \& Yoon, S.-Y. Orexin impairs the phagocytosis and degradation of amyloid- $\beta$ fibrils by microglial cells. J. Alzheimers Dis. 58, 253-261 (2017).

38. Wen, J., Zhao, Y. \& Guo, L. Orexin A induces autophagy in HCT-116 human colon cancer cells through the ERK signaling pathway. Int. J. Mol. Med. 37, 126-132 (2016).

39. Karhu, L., Turku, A. \& Xhaard, H. Modeling of the OX1R-orexin-A complex suggests two alternative binding modes. BMC Struct. Biol. 15, 9. https://doi.org/10.1186/s12900-015-0036-2 (2015).

40. Hilairet, S., Bouaboula, M., Carrière, D., Le Fur, G. \& Casellas, P. Hypersensitization of the Orexin 1 receptor by the CB1 receptor: evidence for cross-talk blocked by the specific CB1 antagonist, SR141716. J. Biol. Chem. 278, 23731-23737. https://doi.org/10.1074/ jbc.M212369200 (2003).

41. Yin, J. et al. Structure and ligand-binding mechanism of the human OX1 and OX2 orexin receptors. Nat. Struct. Mol. Biol. 23, 293-299. https://doi.org/10.1038/nsmb.3183 (2016).

42. Terao, A. et al. Prepro-hypocretin (prepro-orexin) expression is unaffected by short-term sleep deprivation in rats and mice. Sleep 23, 867-874 (2000).

43. Greenbaum, D., Colangelo, C., Williams, K. \& Gerstein, M. Comparing protein abundance and mRNA expression levels on a genomic scale. Genome Biol. 9, 117. https://doi.org/10.1186/gb-2003-4-9-117 (2003).

44. Tse, L. H. \& Wong, Y. H. GPCRs in autocrine and paracrine regulations. Front. Endocrinol. 10, 428. https://doi.org/10.3389/fendo 2019.00428 (2019).

45. Furuse, M. et al. Intracerebroventricular injection of orexins does not stimulate food intake in neonatal chicks. Br. Poult. Sci. 40, 698-700 (1999).

46. Menendez, J. A. \& Lupu, R. Fatty acid synthase and the lipogenic phenotype in cancer pathogenesis. Nat. Rev. Cancer. 7, 763-777 (2007).

47. Kim, K.-H. Regulation of mammalian acetyl-coenzyme A carboxylase. Annu. Rev. Nutr. 17, 77-99 (1997).

48. Wojciechowicz, T. et al. Orexins A and B stimulate proliferation and differentiation of porcine preadipocytes. Exp. Biol. Med. 241, $1786-1795$ (2016).

49. Madison, B. B. Srebp2: A master regulator of sterol and fatty acid synthesis. J. Lipid Res. 57, 333-335 (2016).

50. Wang, Z. et al. Orexin/hypocretin activates mTOR complex 1 (mTORC1) via an Erk/Akt-independent and calcium-stimulated lysosome v-ATPase pathway. J. Biol. Chem. 289, 31950-31959 (2014). 
51. Wang, Y., Viscarra, J., Kim, S.-J. \& Sul, H. S. Transcriptional regulation of hepatic lipogenesis. Nat. Rev. Mol. Cell Biol. 16, 678-689 (2015).

52. Ammoun, S. et al. OX1 orexin receptors activate extracellular signal-regulated kinase in Chinese hamster ovary cells via multiple mechanisms: the role of Ca2+ influx in OX1 receptor signaling. Mol. Endocrinol. 20, 80-99 (2006).

53. Liu, Y., Zhao, Y., Ju, S. \& Guo, L. Orexin A upregulates the protein expression of OX1R and enhances the proliferation of SGC-7901 gastric cancer cells through the ERK signaling pathway. Int. J. Mol. Med. 35, 539-545 (2015).

54. Ramanjaneya, M. et al. Orexin-stimulated MAP kinase cascades are activated through multiple G-protein signalling pathways in human H295R adrenocortical cells: diverse roles for orexins A and B. J. Endocrinol. 202, 249-261 (2009).

55. Ekholm, M. E., Johansson, L. \& Kukkonen, J. P. IP3-independent signalling of OX1 orexin/hypocretin receptors to Ca2+ influx and ERK. Biochem. Biophys. Res. Commun. 353, 475-480. https://doi.org/10.1016/j.bbrc.2006.12.045 (2007).

56. Skrzypski, M. et al. Effects of orexin A on proliferation, survival, apoptosis and differentiation of 3T3-L1 preadipocytes into mature adipocytes. FASEB. 586, 4157-4164 (2012).

57. Roth, G. et al. MAP kinases Erk1/2 phosphorylate sterol regulatory element-binding protein (SREBP)-1a at serine 117 in vitro. J. Biol. Chem. 275, 33302-33307. https://doi.org/10.1074/jbc.M005425200 (2000).

58. Jiao, P., Feng, B., Li, Y., He, Q. \& Xu, H. Hepatic ERK activity plays a role in energy metabolism. Mol. Cell. Endocrinol. 375, 157-166 (2013).

59. Tsai, J., Qiu, W., Kohen-Avramoglu, R. \& Adeli, K. MEK-ERK inhibition corrects the defect in VLDL assembly in HepG2 cells: potential role of ERK in VLDL-ApoB100 particle assembly. Arterioscler. Thromb. Vasc. Biol. 27, 211-218 (2007).

60. Mauvoisin, D., Prévost, M., Ducheix, S., Arnaud, M. P. \& Mounier, C. Key role of the ERK1/2 MAPK pathway in the transcriptional regulation of the Stearoyl-CoA Desaturase (SCD1) gene expression in response to leptin. Mol. Cell. Endocrinol. 319, 116-128 (2010).

61. Yousefi, B. et al. Inhibition of MEK/ERK1/2 signaling affects the fatty acid composition of HepG2 human hepatic cell line. Bioimpacts. 2, 145-150 (2012).

62. Wenzel, J., et al. Hypocretin/orexin increases the expression of steroidogenic enzymes in human adrenocortical NCI H295R cells. Am. J. Physiol.-Reg. Integrative Comp. Physiol. 297, R1601-R1609 (2009).

\section{Acknowledgements}

Authors would like to thank Joshua Flees, Shady Afrin Jessan, and Dr. Alissa Piekarski for their technical assistance.

\section{Author contributions}

S.D. conceived and designed the study. S.D. provided the reagents. E.G., M.Z., T.O., and S.D. conducted the experiments and analyzed the data. S.D. wrote the paper with a critical review by E.G., M.Z., F.S., and T.O.

\section{Funding}

This study was supported by a grant from the Arkansas Bioscience Institute (FY2016-2019, to S.D.).

\section{Competing interests}

The authors declare no competing interests.

\section{Additional information}

Supplementary information is available for this paper at https://doi.org/10.1038/s41598-020-76329-2.

Correspondence and requests for materials should be addressed to S.D.

Reprints and permissions information is available at www.nature.com/reprints.

Publisher's note Springer Nature remains neutral with regard to jurisdictional claims in published maps and institutional affiliations.

(c) (i) Open Access This article is licensed under a Creative Commons Attribution 4.0 International License, which permits use, sharing, adaptation, distribution and reproduction in any medium or format, as long as you give appropriate credit to the original author(s) and the source, provide a link to the Creative Commons licence, and indicate if changes were made. The images or other third party material in this article are included in the article's Creative Commons licence, unless indicated otherwise in a credit line to the material. If material is not included in the article's Creative Commons licence and your intended use is not permitted by statutory regulation or exceeds the permitted use, you will need to obtain permission directly from the copyright holder. To view a copy of this licence, visit http://creativecommons.org/licenses/by/4.0/.

(C) The Author(s) 2020 